\title{
Local Strong Solution for a Class of Shear Thickening Fluids with Non-Newtonian Potential and Heat-Conducting
}

\author{
Yunliang Zhang ${ }^{1}$ and Zhidong Guo ${ }^{2}$ \\ ${ }^{1}$ Institute of Mathematics, Jilin University, Changchun, Jilin 130012, China \\ ${ }^{2}$ Department of Mathematics, Anqing Normal College, Anqing 246133, China \\ Correspondence should be addressed to Yunliang Zhang; zhangyunliang1985@126.com
}

Received 26 June 2015; Revised 1 September 2015; Accepted 13 September 2015

Academic Editor: Luigi C. Berselli

Copyright (c) 2015 Y. Zhang and Z. Guo. This is an open access article distributed under the Creative Commons Attribution License, which permits unrestricted use, distribution, and reproduction in any medium, provided the original work is properly cited.

\begin{abstract}
The aim of this paper is to discuss the model for a class of shear thickening fluids with non-Newtonian potential and heatconducting. Existence and uniqueness of local strong solutions for the model are proved. In this paper, there exist two difficulties we have to overcome. One is the strong nonlinearity of the system. The other is that the state function is not fixed.
\end{abstract}

\section{Introduction}

With the development of the technology, we find that there are a lot of fluids which belong to non-Newtonian fluids in the process of product and nature. The classical non-Newtonian fluids are macromolecule melt and macromolecule liquor and all kinds of slurry and suspend liquor, paint, dope, palette, and biology fluids, for example, in the body of people and animals, the blood, the synovia of arthrosis cavity, lymph liquor, cell liquor, and brain liquor, which are provided with the property of non-Newtonian fluids. So the non-Newtonian fluids exist widely in nature (see [1-6]).

In the absence of gravitational potential term, the mathematical model of non-Newtonian fluids reduces to the Navier-Stokes equations. There have been many results concerning the existence and nonexistence of solutions for such equations (such as [7-10]). In [11], Xin studied the blowup of smooth solutions to the compressible Navier-Stokes equations in arbitrary space dimensions with initial density of compact support. Choe and Kim in $[12,13]$ proved an existence result when $\Omega$ is either a bounded domain or the whole space with the compatibility condition. For other results one can refer to [14-17] and the references cited therein.

In thermodynamics, a state function, function of state, state quantity, or state variable is a property of a system that depends only on the current state of the system, not on the way in which the system acquired that state. A state function describes the equilibrium state of a system. For example, internal energy $e$, temperature $\theta$, density $\rho$, pressure $\pi$, and so on are state quantities because they describe quantitatively an equilibrium state of a thermodynamic system, irrespective of how the system arrived in that state. If two quantities are known, then we can get other quantities. For example, temperature $\theta$ and density $\rho$ are known, and we can get internal energy $e$, pressure $\pi$, and so on.

In mathematics, a state function $z=f(x, y)$ has the following properties:

(i) A state function $z=f(x, y)$ has continuous first partial derivatives.

(ii) There are the tiny variable $d z$ and the limited variable $\int d z$. The integral value depends on the size of the integral upper limit and lower limit and has nothing to do with other factors. Thus,

$$
\int_{1}^{2} d z=f\left(x_{2}, y_{2}\right)-f\left(x_{1}, y_{1}\right)=z_{2}-z_{1}
$$

(iii) Consider

$$
\frac{\partial^{2} z}{\partial x \partial y}=\frac{\partial^{2} z}{\partial y \partial x} .
$$

The following are some examples of state equations:

(i) Polytropic gases: $\phi=A \rho^{\gamma}$ ( $A$ is a constant and $\gamma$ is gas thermal insulation coefficient and is a constant) (see [15-17]). 
(ii) State equation of ideal gas: $\psi=R \rho \theta$ ( $R$ is a constant) (see [18]).

(iii) Isentropic process: $\varphi=\theta$ (see [18]).

In this paper, we consider generalized state equation. Energy increases with the increase of temperature. In mathematics, $\pi=\varphi(\theta, \rho)$ and $\varphi_{1}=\partial \varphi / \partial \theta>0$ (we define $\varphi_{1}=\partial \varphi / \partial \theta, \varphi_{2}=\partial \varphi / \partial \rho, \psi_{1}=\partial \psi / \partial \theta$, and $\left.\psi_{2}=\partial \psi / \partial \rho\right)$. Ignore the molecular potential energy and all of the gas fluid, for example, methane, propane, and butane from the oil.

In this paper, we consider a class of heat-conducting nonNewtonian fluids of Navier-Stokes system:

$$
\begin{aligned}
& \rho_{t}+(\rho u)_{x}=0, \quad(x, t) \in \Omega_{T}, \\
& (\rho e)_{t}+(\rho u e)_{x}-\left[\left(\theta_{x}^{2}+\mu\right)^{(p-2) / 2} \theta_{x}\right]_{x}+\pi u_{x}=\left|u_{x}\right|^{2}, \\
& (\rho u)_{t}+\left(\rho u^{2}\right)_{x}-\left[\left(u_{x}^{2}+\mu_{2}\right)^{(q-2) / 2} u_{x}\right]_{x}+\Omega_{T}, \\
& e=\varphi(\theta, \rho), \quad(x, t) \in \Omega_{T}, \\
& \pi=\psi(\theta, \rho), \quad(x, t) \in \Omega_{T},
\end{aligned}
$$

with the initial and boundary conditions

$$
\begin{aligned}
\left.(\rho, u, \theta)\right|_{t=0} & =\left(\rho_{0}, u_{0}, \theta_{0}\right), \quad x \in[0,1], \\
u(0, t) & =u(1, t)=0, \quad t \in[0, T], \\
\theta(0, t) & =\theta(1, t)=0, \quad t \in[0, T],
\end{aligned}
$$

where $\Omega_{T}=I \times(0, T), I=(0,1)$, the initial density $\rho_{0} \geq 0$, $p, q>2$, and $\mu_{1}, \mu_{2}>0$ are given constants. The unknown variables $\rho, u$, and $\theta$ denote the density, velocity, temperature, and the non-Newtonian gravitational potential, respectively.

Definition 1. The triple $(\rho, u, \theta)$ is called a strong solution to the initial boundary value problem (3)-(4), if the following conditions are satisfied:

(i) Consider

$$
\begin{gathered}
0 \leq \rho \in C\left([0, T] ; H^{1}(I)\right), \\
u, \theta \in C\left([0, T] ; H_{0}^{1}(I)\right) \cap L^{\infty}\left(0, T ; H^{2}(I)\right), \\
\rho_{t} \in C\left([0, T] ; L^{2}(I)\right), \\
u_{t}, \theta_{t} \in L^{2}\left(0, T ; H_{0}^{1}(I)\right), \\
\sqrt{\rho} u_{t}, \sqrt{\rho} \theta_{t} \in L^{\infty}\left(0, T ; L^{2}(I)\right) .
\end{gathered}
$$

(ii) For all $\chi \in C\left([0, T] ; H^{1}(I)\right), \chi_{t} \in L^{\infty}\left(0, T ; L^{2}(I)\right)$, for a.e. $t \in(0, T)$, we have

$$
\begin{aligned}
& \int_{0}^{1} \rho \chi(x, t) d x-\int_{0}^{t} \int_{0}^{1}\left(\rho \chi_{t}+\rho u \chi_{x}\right)(x, s) d x d s \\
& \quad=\int_{0}^{1} \rho_{0} \chi(x, 0) d x .
\end{aligned}
$$

(iii) For all $\phi \in C\left([0, T] ; H_{0}^{1}(I)\right) \cap L^{\infty}\left(0, T ; H^{2}(I)\right), \phi_{t} \in$ $L^{2}\left(0, T ; H_{0}^{1}(I)\right)$, for a.e. $t \in(0, T)$, we have

$$
\begin{aligned}
& \int_{0}^{1} \operatorname{\rho e\phi }(x, t) d x-\int_{0}^{t} \int_{0}^{1}\left(\rho e \phi_{t}\right. \\
& \left.\quad+\left(\rho u e-\left(\theta_{x}^{2}+\mu_{1}\right)^{(p-2) / 2} \theta_{x}\right) \phi_{x}\right)(x, s) d x d s \\
& \quad+\int_{0}^{t} \int_{0}^{1}-\left(\left|u_{x}\right|^{2}+\pi u_{x}\right) \phi(x, s) d x d s \\
& \quad=\int_{0}^{1} \rho_{0} e_{0} \phi(x, 0) d x .
\end{aligned}
$$

(iv) For all $\phi \in C\left([0, T] ; H_{0}^{1}(I)\right) \cap L^{\infty}\left(0, T ; H^{2}(I)\right), \phi_{t} \in$ $L^{2}\left(0, T ; H_{0}^{1}(I)\right)$, for a.e. $t \in(0, T)$, we have

$$
\begin{aligned}
& \int_{0}^{1} \rho u \phi(x, t) d x-\int_{0}^{t} \int_{0}^{1}\left\{\rho u \phi_{t}+\rho u^{2} \phi_{x}\right. \\
& \left.-\left(u_{x}^{2}+\mu_{2}\right)^{(q-2) / 2} u_{x} \phi_{x}+\pi_{x} \phi\right\}(x, s) d x d s \\
& \quad=\int_{0}^{1} \rho_{0} u_{0} \phi(x, 0) d x .
\end{aligned}
$$

\subsection{Main Results}

Theorem 2. Assume that $\left(\rho_{0}, u_{0}, \theta_{0}\right)$ satisfies the following conditions:

$$
\begin{aligned}
& 0<\delta \leq \rho_{0} \in H^{1}(I), u_{0}, \theta_{0} \in H_{0}^{1}(I) \cap H^{2}(I), \\
& 0<\delta \leq \varphi_{1}
\end{aligned}
$$

and further that there exists a constant $c_{0}>0$, such that

$$
1+\left|\rho_{0}\right|_{H^{1}}+\left|\theta_{0}\right|_{H^{1}}+\left|u_{0}\right|_{H^{1}} \leq c_{0} .
$$

Then there exist a time $T^{*} \in(0,+\infty)$ and a unique strong solution $(\rho, u, \theta)$ to (3)-(4) such that

$$
\begin{array}{r}
\rho \in C\left(\left[0, T^{*}\right] ; H^{1}(I)\right), \\
\rho_{t} \in C\left(\left[0, T^{*}\right] ; L^{2}(I)\right), \\
u_{t}, \theta_{t} \in L^{2}\left(0, T^{*} ; H_{0}^{1}(I)\right), \\
u, \theta \in C\left(\left[0, T^{*}\right] ; H_{0}^{1}(I)\right) \\
\cap t^{\infty}\left(0, T^{*} ; H^{2}(I)\right), \\
\sqrt{\rho} u_{t}, \sqrt{\rho} \theta_{t} \in L^{\infty}\left(0, T^{*} ; L^{2}(I)\right) .
\end{array}
$$

The rest of this paper is organized as follows. In Section 2, we present some elementary lemmas. In Section 3, we devote ourselves to the study of the problem with positive density. In Section 4 we give the proof of the main theorem. 


\section{Preliminaries}

We first give some known facts for later use.

Lemma 3 (see [15]). Assume that $f=0$ on $\partial \Omega$, where $\Omega \subset R^{1}$ is bounded and open, $f \in C^{2+\alpha}(\bar{\Omega}), 0<\alpha<1$. Then

$$
\left|f^{\prime}\right|_{L^{\infty}(\Omega)} \leq d^{1 / 2}(\Omega)\left|f^{\prime \prime}\right|_{L^{2}(\Omega)},
$$

where $d(\Omega)$ denotes the length of $\Omega$.

Lemma 4 (see [15]). Let $H$ be a Hilbert space with a scalar product $(\cdot, \cdot)_{H}$ and let $X$ be a Banach space such that

$$
X \hookrightarrow H \cong H^{*} \hookrightarrow X^{*},
$$

and $X$ is dense in $H, p>1$. Then

$$
W \equiv\left\{u \in L^{p}(I ; X) ; \frac{d u}{d t} \in L^{p^{\prime}}\left(I ; X^{*}\right)\right\} \hookrightarrow C(I ; H) .
$$

Lemma 5. $\pi=\varphi(\theta, \rho)$ and $e=\phi(\theta, \rho)$ is state function. According to the mathematical properties of state function, we can get that $\exists M>0$,

$$
\begin{gathered}
|\varphi|+\left|\varphi_{1}\right|+\left|\varphi_{2}\right|+\left|\varphi_{11}\right|+\left|\varphi_{22}\right| \leq M, \\
|\psi|+\left|\psi_{1}\right|+\left|\psi_{2}\right|+\left|\psi_{11}\right|+\left|\psi_{22}\right| \leq M .
\end{gathered}
$$

\section{Existence of Solutions with Positive Density}

3.1. A Priori Estimates for Smooth Solutions. We first construct approximate solutions of problem (3)-(4). By using the iterative scheme, inductively, consider the following:

(i) Firstly define $u^{0}=0, \theta^{0}=0$.

(ii) Assuming that $u^{k-1}, \theta^{k-1}$ were defined for $k \geq 1$, we can obtain $\left(\rho^{k}, u^{k}, \theta^{k}, e^{k}, \pi^{k}\right)$ as the unique smooth solution to the following system:

$$
\begin{aligned}
& \rho_{t}^{k}+u^{k-1} \rho_{x}^{k}+u_{x}^{k-1} \rho^{k}=0, \quad(x, t) \in \Omega_{T}, \\
& \pi^{k}=\phi\left(\theta^{k}, \rho^{k}\right), \quad(x, t) \in \Omega_{T}, \\
& e^{k}=\varphi\left(\theta^{k}, \rho^{k}\right), \quad(x, t) \in \Omega_{T}, \\
& \rho^{k} \varphi_{1}^{k} \theta_{t}^{k}+\rho^{k} \varphi_{1}^{k} u^{k-1} \theta_{x}^{k}-\left(\rho^{k}\right)^{2} \varphi_{2}^{k} u_{x}^{k-1}+H_{p}^{\mu_{1}} \theta^{k} \\
& \quad+\psi^{k} u_{x}^{k-1}-\left(u_{x}^{k-1}\right)^{2}=0, \quad(x, t) \in \Omega_{T}, \\
& \rho^{k} u_{t}^{k}+\rho^{k} u^{k-1} u_{x}^{k}-H_{q}^{\mu_{2}} \theta^{k}+\psi_{x}=0, \quad(x, t) \in \Omega_{T}, \\
& \rho^{k}(x, 0)=\rho_{0}, \\
& u^{k}(x, 0)=u_{0}, \\
& \theta^{k}(x, 0)=\theta_{0}, \\
& u^{k}(0, t)=u^{k}(1, t)=0, \\
& \theta^{k}(0, t)=\theta^{k}(1, t)=0,
\end{aligned}
$$

where $m_{0}=\int_{0}^{1} \rho_{0}(x) d x>0$ is the initial mass, for a given function $\omega$ and numbers $s>2, \mu>0$,

$$
H_{s}^{\mu} \omega=-\left[\left(\left(\omega_{x}\right)^{2}+\mu\right)^{(s-2) / 2} \omega_{x}\right]_{x}
$$

With this process, the nonlinear coupled system has been deduced into a sequence of decoupled problems and each problem admits a smooth solution. Moreover, we find $\rho^{k}$ from (16), (21) with the smooth function $u^{k-1}$; that is,

$$
\begin{aligned}
\rho_{t}^{k}+u^{k-1} \rho_{x}^{k-1}+u_{x}^{k-1} \rho^{k-1} & =0, \\
\left.\rho^{k}\right|_{t=0} & =\rho_{0} .
\end{aligned}
$$

Obviously there is a unique solution $\rho^{k}$ to the above initial value problem and also by a standard argument, we could get

$$
\rho^{k}(x, t) \geq \delta \exp \left(-\int_{0}^{T^{*}}\left|u_{x}^{k-1}(\cdot, s)\right|_{L^{\infty}} d s\right)>0,
$$

$$
\forall t \in\left(0, T^{*}\right)
$$

Then by (19) and (21) we get $\theta^{k}$. Lastly, with $\rho^{k}, \theta^{k}$, we obtain $u^{k}$ from (20) and (21).

Moreover, assume that for a given $T_{1}, 0<T_{1} \leq T$

$$
\begin{array}{r}
\sup _{0 \leq t \leq T_{1}}\left|u_{x x}^{k-1}\right|_{L^{2}}+\int_{0}^{T_{1}}\left|u_{x t}^{k-1}\right|_{L^{2}}^{2} d s \leq c_{1}, \\
\sup _{0 \leq t \leq T_{1}}\left|u_{x x}^{k-1}\right|_{L^{2}} \leq c_{2} .
\end{array}
$$

For some, $c_{2}>c_{1}>c_{0}>1$ will be fixed later. We will prove that

$$
\begin{array}{r}
\sup _{0 \leq t \leq T_{1}}\left|u_{x}^{k}\right|_{L^{2}}+\int_{0}^{T_{1}}\left|u_{x t}^{k}\right|_{L^{2}}^{2} d s \leq c_{1}, \\
\sup _{0 \leq t \leq T_{1}}\left|u_{x x}^{k}\right|_{L^{2}} \leq c_{2},
\end{array}
$$

and some more useful uniform estimate about $\left(\rho^{k}, u^{k}, \theta^{k}\right)$.

(i) Estimation for $\rho^{k}$.

Then multiplying (16) by $\rho^{k}$ and integrating it over $(0,1)$ with respect to $x$, we have

$$
\frac{1}{2} \frac{d}{d t} \int_{0}^{1}\left|\rho^{k}\right|^{2} d s+\int_{0}^{1}\left(\rho^{k-1} u^{k-1}\right)_{x} \rho^{k} d x=0
$$

Integrating by parts, using the Sobolev inequality and Lemma 3, we deduce that

$$
\frac{d}{d t}\left|\rho^{k}(t)\right|_{L^{2}}^{2} \leq\left|u_{x x}^{k-1}\right|_{L^{2}}\left|\rho^{k}\right|_{L^{2}}^{2}
$$


4

Advances in Mathematical Physics

Then, considering differential equation (16) with respect to $x$ and multiplying it by $\rho_{x}^{k}$, integrating it over $(0,1)$ on $x$, and using the Sobolev inequality, we have

$$
\begin{aligned}
& \frac{d}{d t} \int_{0}^{1}\left|\rho_{x}^{k}\right|^{2} d x \\
& \quad=-\int_{0}^{1}\left[\frac{3}{2} u_{x}^{k-1}\left(\rho_{x}^{k}\right)^{2}+\rho^{k} \rho_{x}^{k} u_{x x}^{k-1}\right](t) d x \\
& \quad \leq \frac{3}{2}\left[\left|u_{x}^{k-1}\right|_{L^{\infty}}\left|\rho_{x}^{k}\right|_{L^{2}}^{2}+\left|\rho^{k}\right|_{L^{\infty}}\left|u_{x x}^{k-1}\right|_{L^{2}}\right] \\
& \quad \leq 3\left|\rho_{x}^{k}\right|_{L^{2}}^{2}\left|u_{x x}^{k-1}\right|_{L^{2}} .
\end{aligned}
$$

From (28) and (29), by Gronwall's inequality, it follows that

$$
\sup _{0 \leq t \leq T}\left|\rho^{k}(t)\right|_{H^{1}}^{2} \leq\left|\rho_{0}^{k}\right|_{H^{1}}^{2} \exp \left\{4 \int_{0}^{t}\left|u_{x x}^{k-1}\right|_{L^{2}} d s\right\},
$$

and, taking $T_{2}=c_{2}^{-1}$, we get

$$
\begin{aligned}
\sup _{0 \leq t \leq T}\left|\rho^{k}(t)\right|_{H^{1}} & \leq\left|\rho_{0}^{k}\right|_{H^{1}}\left[\exp \left\{4 \int_{0}^{t}\left|u_{x x}^{k-1}\right|_{L^{2}} d s\right\}\right]^{1 / 2} \\
& \leq C c_{0} .
\end{aligned}
$$

And using (16) we can also obtain

$$
\left|\rho_{t}^{k}(t)\right|_{L^{2}} \leq\left|\rho_{x}^{k-1}\right|_{L^{2}}\left|u_{x}^{k-1}\right|_{L^{2}}+\left|\rho^{k-1}\right|_{L^{2}}\left|u_{x x}^{k-1}\right|_{L^{2}} \leq C c_{1}^{2},
$$

where $C$ is a positive constant.

(ii) Estimation for $\theta^{k}(t)$.

Differentiating (19) with respect to $t$, multiplying it by $\theta_{t}^{k}$, and integrating it over $(0,1)$ on $x$, we derive

$$
\begin{aligned}
& \frac{1}{2} \frac{d}{d t} \int_{0}^{1} \rho^{k} \varphi_{1}^{k}\left|\theta_{t}^{k}\right|^{2} d x \\
& \quad+\int_{0}^{1}\left[\left(\theta_{x}^{2}+\mu_{1}\right)^{(p-2) / 2} \theta_{x}\right]_{t} \theta_{x t}^{k} d x \\
& \quad=\frac{1}{2} \int_{0}^{1}\left[\rho^{k} \varphi_{1}^{k}\right]_{t}\left|\theta_{t}^{k}\right|^{2} d x-\int_{0}^{1}\left[\rho^{k} \varphi_{1}^{k} u^{k-1} \theta_{x}^{k}\right]_{t} \theta_{t}^{k} d x \\
& \quad-\int_{0}^{1}\left[\left(\rho^{k}\right)^{2} \varphi_{2}^{k} u_{x}^{k-1}\right]_{t} \theta_{t}^{k} d x-\int_{0}^{1}\left[\psi^{k} u_{x}^{k-1}\right]_{t} \theta_{t}^{k} d x \\
& \quad+\int_{0}^{1}\left(u_{x}^{k-1}\right)_{t}^{2} \theta_{t}^{k} d x=\sum_{j=1}^{5} I_{j} .
\end{aligned}
$$

Using the Sobolev inequality, Young's inequality, Lemma 3, and (25), (30) and (31), we obtain

$$
\begin{aligned}
I_{1}= & \frac{1}{2} \int_{0}^{1}\left[\rho^{k} \varphi_{1}^{k}\right]_{t}\left|\theta_{t}^{k}\right|^{2} d x \\
= & \frac{1}{2} \int_{0}^{1} \rho_{t}^{k} \varphi_{1}^{k}\left|\theta_{t}^{k}\right|^{2} d x+\frac{1}{2} \int_{0}^{1} \rho^{k} \varphi_{11}^{k} \theta_{t}^{k}\left|\theta_{t}^{k}\right|^{2} d x \\
& +\frac{1}{2} \int_{0}^{1} \rho^{k} \varphi_{12}^{k} \rho_{t}^{k}\left|\theta_{t}^{k}\right|^{2} d x=\sum_{j=1}^{3} I_{1 j} .
\end{aligned}
$$

Here,

$$
\begin{aligned}
& I_{11}=\frac{1}{2} \int_{0}^{1} \rho_{t}^{k} \varphi_{1}^{k}\left|\theta_{t}^{k}\right|^{2} d x \leq\left. C\left|\int_{0}^{1} \rho_{t}^{k}\right| \theta_{t}^{k}\right|^{2} d x \mid \\
& \leq C\left|\int_{0}^{1} \rho^{k} u^{k-1} \theta_{t}^{k} \theta_{x t}^{k} d x\right| \\
& \leq C\left|\rho^{k}\right|_{L^{\infty}}^{1 / 2}\left|\sqrt{\rho^{k}} \theta_{t}^{k}\right|_{L^{2}}\left|u^{k-1}\right|_{L^{\infty}}\left|\theta_{x t}^{k}\right|_{L^{2}} \\
& \leq C c_{0}^{1 / 2} c_{1}\left|\sqrt{\rho^{k}} \theta_{t}^{k}\right|_{L^{2}}\left|\theta_{x t}^{k}\right|_{L^{2}} \\
& \leq \eta\left|\theta_{x t}^{k}\right|_{L^{2}}^{2}+C_{\eta} c_{1}^{3}\left|\sqrt{\rho^{k}} \theta_{t}^{k}\right|_{L^{2}}^{2}, \\
& I_{12}=\frac{1}{2} \int_{0}^{1} \rho^{k} \varphi_{11}^{k} \theta_{t}^{k}\left|\theta_{t}^{k}\right|^{2} d x \leq\left. C\left|\int_{0}^{1} \rho^{k} \theta_{t}^{k}\right| \theta_{t}^{k}\right|^{2} d x \mid \\
& \leq C\left|\theta_{t}^{k}\right|_{L^{\infty}}\left|\sqrt{\rho^{k}} \theta_{t}^{k}\right|_{L^{2}}^{2} \leq \eta\left|\theta_{x t}^{k}\right|_{L^{2}}^{2}+C_{\eta}\left|\sqrt{\rho^{k}} \theta_{t}^{k}\right|_{L^{2}}^{4}, \\
& I_{13}=\frac{1}{2} \int_{0}^{1} \rho^{k} \varphi_{12}^{k-1} \rho_{t}^{k}\left|\theta_{t}^{k}\right|^{2} d x \\
& \leq\left. C\left|\rho^{k}\right|_{L^{\infty}}\left|\int_{0}^{1} \rho_{t}^{k}\right| \theta_{t}^{k}\right|^{2} d x \mid \\
& \leq C c_{0}^{3 / 2} c_{1}\left|\sqrt{\rho^{k}} \theta_{t}^{k}\right|_{L^{2}}\left|\theta_{x t}^{k}\right|_{L^{2}} \\
& \leq \eta\left|\theta_{x t}^{k}\right|_{L^{2}}^{2}+C_{\eta} c_{1}^{5}\left|\sqrt{\rho^{k}} \theta_{t}^{k}\right|_{L^{2}}^{2} .
\end{aligned}
$$

In the same way, we have

$$
\begin{aligned}
I_{2}= & -\int_{0}^{1}\left[\rho^{k} \varphi_{1}^{k} u^{k-1} \theta_{x}^{k}\right]_{t} \theta_{t}^{k} d x \\
= & -\int_{0}^{1} \rho_{t}^{k} \varphi_{1}^{k} u^{k-1} \theta_{x}^{k} \theta_{t}^{k} d x-\int_{0}^{1} \rho^{k} \varphi_{11}^{k} \theta_{t}^{k} u^{k-1} \theta_{x}^{k} \theta_{t}^{k} d x \\
& -\int_{0}^{1} \rho^{k} \varphi_{12}^{k} \rho_{t}^{k} u^{k} \theta_{x}^{k} \theta_{t}^{k} d x-\int_{0}^{1} \rho^{k} \varphi_{1}^{k} u_{t}^{k-1} \theta_{x}^{k} \theta_{t}^{k} d x \\
& -\int_{0}^{1} \rho^{k} \varphi_{1}^{k} u^{k-1} \theta_{x t}^{k} \theta_{t}^{k} d x=\sum_{j=1}^{5} I_{2 j} .
\end{aligned}
$$

Here,

$$
\begin{aligned}
I_{21} & =-\int_{0}^{1} \rho_{t}^{k} \varphi_{1}^{k} u^{k-1} \theta_{x}^{k} \theta_{t}^{k} d x \leq C\left|\int_{0}^{1} \rho_{t}^{k} u^{k-1} \theta_{x}^{k} \theta_{t}^{k} d x\right| \\
& \leq C\left|\rho_{t}^{k}\right|_{L^{2}}\left|u^{k-1}\right|_{L^{\infty}}\left|\theta_{x}^{k}\right|_{L^{2}}\left|\theta_{t}^{k}\right|_{L^{\infty}} \\
& \leq C\left|\rho_{t}^{k}\right|_{L^{2}}\left|u_{x}^{k-1}\right|_{L^{2}}\left|\theta_{x}^{k}\right|_{L^{2}}\left|\theta_{x t}^{k}\right|_{L^{2}} \leq C c_{1}^{3}\left|\theta_{x}^{k}\right|_{L^{2}}\left|\theta_{x t}^{k}\right|_{L^{2}} \\
& \leq \eta\left|\theta_{x t}^{k}\right|_{L^{2}}^{2}+C_{\eta} c_{1}^{6}\left|\theta_{x}^{k}\right|_{L^{2}}^{2},
\end{aligned}
$$


Advances in Mathematical Physics

5

$$
\begin{aligned}
I_{22} & =-\int_{0}^{1} \rho^{k} \varphi_{11}^{k} \theta_{t}^{k} u^{k-1} \theta_{x}^{k} \theta_{t}^{k} d x \\
& \leq C\left|\int_{0}^{1} \rho^{k} \theta_{t}^{k} u^{k-1} \theta_{x}^{k} \theta_{t}^{k} d x\right| \\
& \leq C\left|\rho^{k}\right|_{L^{\infty}}^{1 / 2}\left|u^{k-1}\right|_{L^{\infty}}\left|\theta_{t}^{k}\right|_{L^{\infty}}\left|\theta_{x}^{k}\right|_{L^{2}}\left|\sqrt{\rho^{k}} \theta_{t}^{k}\right|_{L^{2}} \\
& \leq \eta\left|\theta_{x x}^{k}\right|_{L^{2}}^{2}+C_{\eta} c_{1}^{3}\left|\theta_{x}^{k}\right|_{L^{2}}^{2}\left|\sqrt{\rho^{k}} \theta_{t}^{k}\right|_{L^{2}}^{2} \\
I_{23} & =-\int_{0}^{1} \rho^{k} \varphi_{12}^{k} \rho_{t}^{k} u^{k-1} \theta_{x}^{k} \theta_{t}^{k} d x \\
& \leq C\left|\int_{0}^{1} \rho^{k} \rho_{t}^{k} u^{k-1} \theta_{x}^{k} \theta_{t}^{k} d x\right| \\
& \leq C\left|\rho^{k}\right|_{L^{\infty}}\left|\rho_{t}^{k}\right|_{L^{2}}\left|u^{k-1}\right|_{L^{\infty}}\left|\theta_{x}^{k}\right|_{L^{2}}\left|\theta_{t}^{k}\right|_{L^{\infty}} \\
& \leq \eta\left|\theta_{x t}^{k}\right|_{L^{2}}^{2}+C_{\eta} c_{1}^{8}\left|\theta_{x}^{k}\right|_{L^{2}}^{2}, \\
& \leq C\left|\rho^{k}\right|_{L^{\infty}}^{1 / 2}\left|\sqrt{\rho^{k}} \theta_{t}^{k}\right|_{L^{2}}\left|u^{k-1}\right|_{L^{\infty}}\left|\theta_{x t}^{k}\right|_{L^{2}} \\
& \leq \eta\left|\theta_{x t}^{k}\right|_{L^{2}}^{2}+C_{\eta} c_{1}^{6}\left|\sqrt{\rho^{k}} \theta_{t}^{k}\right|_{L^{2}}^{2} \\
& \leq C\left|\int_{0}^{1} \rho^{k} \varphi_{1}^{k} u_{t}^{k-1} \theta_{x}^{k} \theta_{t}^{k} d x \leq C\right| \int_{L^{\infty}}^{1 / 2}\left|\sqrt{\rho^{k}} \theta_{t}^{k}\right|_{L^{2}}^{k}\left|u_{t}^{k-1}\right|_{L^{\infty}}^{k-1} \theta_{x}^{k} \theta_{t}^{k} d x \mid \\
& \leq C c_{0}^{k}\left|\theta_{x}^{k}\right|_{L^{2}}^{2}\left|\sqrt{\rho^{k}} \theta_{t}^{k}\right|_{L^{2}}^{2}+\frac{1}{2}\left|u_{x t}^{k-1}\right|_{L^{2}}^{2}, \\
I_{25} & =-\int_{0}^{1} \rho^{k} \varphi_{1}^{k} u^{k-1} \theta_{x t}^{k} \theta_{t}^{k} d x \\
& =C \rho_{\rho^{k}}^{k} \theta_{t}^{k}\left|u^{k-1}\right|_{L^{\infty}}\left|\theta_{x t}^{k}\right|_{L^{2}} \\
I^{2} &
\end{aligned}
$$

Following the same method, we obtain

$$
\begin{aligned}
I_{3}= & -\int_{0}^{1}\left[\left(\rho^{k}\right)^{2} \varphi_{2}^{k} u_{x}^{k-1}\right]_{t} \theta_{t}^{k} d x \\
= & -\int_{0}^{1} 2 \rho^{k} \rho_{t}^{k} \varphi_{2}^{k} u_{x}^{k-1} \theta_{t}^{k} d x \\
& -\int_{0}^{1}\left(\rho^{k}\right)^{2} \varphi_{12}^{k} \theta_{t}^{k} u_{x}^{k-1} \theta_{t}^{k} d x \\
& -\int_{0}^{1}\left(\rho^{k}\right)^{2} \varphi_{22}^{k} \rho_{t}^{k} u_{x}^{k-1} \theta_{t}^{k} d x \\
& -\int_{0}^{1}\left(\rho^{k}\right)^{2} \varphi_{2}^{k} u_{x t}^{k-1} \theta_{t}^{k} d x=\sum_{j=1}^{4} I_{3 j} .
\end{aligned}
$$

Here,

$$
\begin{aligned}
I_{31} & =-\int_{0}^{1} 2 \rho^{k} \rho_{t}^{k} \varphi_{2}^{k} u_{x}^{k-1} \theta_{t}^{k} d x \\
& \leq C\left|\int_{0}^{1} \rho^{k} \rho_{t}^{k} u_{x}^{k-1} \theta_{t}^{k} d x\right| \\
& \leq\left. C\left|\rho^{k}\right|_{L^{\infty}}\left|\rho_{t}^{k}\right|\right|_{L^{2}}\left|u_{x}^{k-1}\right|_{L^{2}}\left|\theta_{t}^{k}\right|_{L^{\infty}} \\
& \leq \eta\left|\theta_{x x}^{k}\right|_{L^{2}}^{2}+C_{\eta} c_{1}^{8}, \\
I_{32} & =-\int_{0}^{1}\left(\rho^{k}\right)^{2} \varphi_{12}^{k} \theta_{t}^{k} u_{x}^{k-1} \theta_{t}^{k} d x \\
& \leq C\left|\int_{0}^{1}\left(\rho^{k}\right)^{2} \theta_{t}^{k} u_{x}^{k-1} \theta_{t}^{k} d x\right| \\
& \leq C\left|\rho^{k}\right|_{L^{\infty}}\left|u_{x}^{k-1}\right|_{L^{\infty}}\left|\sqrt{\rho^{k}} \theta_{t}^{k}\right|_{L^{2}}^{2} \leq C_{\eta} c_{1}^{2}\left|\sqrt{\rho^{k}} \theta_{t}^{k}\right|_{L^{2}}^{2} \\
I_{33} & =-\int_{0}^{1}\left(\rho^{k}\right)^{2} \varphi_{22}^{k} \rho_{t}^{k} u_{x}^{k-1} \theta_{t}^{k} d x \\
& \leq C\left|\int_{0}^{1}\left(\rho^{k}\right)^{2} \rho_{t}^{k} u_{x}^{k-1} \theta_{t}^{k} d x\right| \\
& \leq C\left|\int_{0}^{1}\left(\rho^{k}\right)^{2} u_{x t}^{k-1} \theta_{t}^{k} d x\right| \\
& \leq C\left|\rho^{k}\right|_{L^{\infty}}^{2}\left|\rho_{t}^{k}\right|_{L^{2}}\left|u_{x}^{k-1}\right|_{L^{2}}\left|\theta_{t}^{k}\right|_{L^{\infty}}^{2}\left|u_{x t}^{k-1}\right| \theta_{L^{2}}\left|\theta_{t}^{k}\right|_{L^{\infty}} \\
& \leq \eta\left|\theta_{x t}^{k}\right|_{L^{2}}^{2}+C_{\eta} c_{L^{2}}^{10}+C_{\eta} c_{1}^{4}\left|u_{x t}^{k-1}\right|_{L^{2}}^{2} \\
I_{34} & =-\int_{0}^{1}\left(\rho^{k}\right)^{2} \varphi_{2}^{k} u_{x t}^{k-1} \theta_{t}^{k} d x \\
& \\
&
\end{aligned}
$$

In a similar way,

$$
\begin{aligned}
I_{4}= & -\int_{0}^{1}\left[\psi^{k} u_{x}^{k-1}\right]_{t} \theta_{t}^{k} d x \\
= & -\int_{0}^{1} \psi_{1}^{k} \theta_{t}^{k} u_{x}^{k-1} \theta_{t}^{k} d x-\int_{0}^{1} \psi_{2}^{k} \rho_{t}^{k} u_{x}^{k-1} \theta_{t}^{k} d x \\
& -\int_{0}^{1} \psi^{k-1} u_{x t}^{k-1} \theta_{t}^{k} d x=\sum_{j=1}^{3} I_{4 j} .
\end{aligned}
$$


6

Advances in Mathematical Physics

Here,

$$
\begin{aligned}
I_{41} & =-\int_{0}^{1} \psi_{1}^{k} \theta_{t}^{k} u_{x}^{k-1} \theta_{t}^{k} d x \leq C\left|\int_{0}^{1} \frac{\rho^{k}}{\rho^{k}} \theta_{t}^{k} u_{x}^{k-1} \theta_{t}^{k} d x\right| \\
& \leq C\left|u_{x}^{k-1}\right|_{L^{\infty}}\left|\sqrt{\rho^{k}} \theta_{t}^{k}\right|_{L^{2}}^{2} \leq C c_{1}\left|\sqrt{\rho^{k}} \theta_{t}^{k}\right|_{L^{2}}^{2}, \\
I_{42} & =-\int_{0}^{1} \psi_{2}^{k} \rho_{t}^{k} u_{x}^{k-1} \theta_{t}^{k} d x \leq C\left|\int_{0}^{1} \rho_{t}^{k} u_{x}^{k-1} \theta_{t}^{k} d x\right| \\
& \leq C\left|\rho_{t}^{k}\right|_{L^{2}}\left|u_{x}^{k-1}\right|_{L^{2}}\left|\theta_{t}^{k}\right|_{L^{\infty}} \leq \eta\left|\theta_{x t}^{k}\right|_{L^{2}}^{2}+C_{\eta} c_{1}^{10}, \\
I_{43} & =-\int_{0}^{1} \psi^{k} u_{x t}^{k-1} \theta_{t}^{k} d x \leq C\left|\int_{0}^{1} u_{x t}^{k-1} \theta_{t}^{k} d x\right| \\
& \leq C\left|u_{x t}^{k-1}\right|_{L^{2}}\left|\theta_{t}^{k}\right|_{L^{\infty}} \leq \eta\left|\theta_{x t}^{k}\right|_{L^{2}}^{2}+C_{\eta} c_{1}^{2} .
\end{aligned}
$$

Similarly,

$$
\begin{aligned}
I_{5} & =\int_{0}^{1}\left(u_{x}^{k-1}\right)_{t}^{2} \theta_{t}^{k} d x \leq 2\left|u_{x}^{k-1}\right|_{L^{\infty}}\left|u_{x t}^{k-1}\right|_{L^{2}}\left|\theta^{k}\right|_{L^{\infty}} \\
& \leq 2\left|u_{x x}^{k-1}\right|_{L^{2}}\left|u_{x t}^{k-1}\right|_{L^{2}}\left|\theta_{x t}^{k}\right|_{L^{2}} \\
& \leq \eta\left|\theta_{x t}^{k}\right|_{L^{2}}^{2}+C_{\eta} c_{1}^{2}\left|u_{x t}^{k-1}\right|_{L^{2}}^{2}
\end{aligned}
$$

Substituting $I_{j}(j=1,2, \ldots, 5)$ into (43), after choosing $\eta=\mu_{1}^{(p-2) / 2} / 26$, we get

$$
\begin{gathered}
\frac{1 \frac{d}{2} d t}{d}\left|\sqrt{\rho^{k} \varphi_{1}^{k}} \theta_{t}^{k}(t)\right|_{L^{2}}^{2}+\frac{1}{2} \mu_{1}^{(p-2) / 2}\left|\theta_{x t}^{k}\right|_{L^{2}}^{2}(s) d s \\
\leq C c_{1}^{10}+C c_{1}^{8}\left|\sqrt{\rho^{k}} \theta_{t}^{k}\right|_{L^{2}}^{2}+C\left|\sqrt{\rho^{k}} \theta_{t}^{k}\right|_{L^{2}}^{4} \\
+C c_{1}^{6}\left|\theta_{x}^{k}\right|_{L^{2}}^{2}+C c_{1}^{3}\left|\theta_{x}^{k}\right|_{L^{2}}^{2}\left|\sqrt{\rho^{k}} \theta_{t}^{k}\right|_{L^{2}}^{2} \\
+C c_{1}^{4}\left|u_{x t}^{k-1}\right|_{L^{2}}^{2},
\end{gathered}
$$

and, integrating it over $(\tau, t) \subset(0, T)$, we have

$$
\begin{aligned}
\frac{1}{2} \mid & \left.\sqrt{\rho^{k} \varphi_{1}^{k}} \theta_{t}^{k}(t)\right|_{L^{2}} ^{2}+\frac{1}{2} \mu_{1}^{(p-2) / 2} \int_{\tau}^{t}\left|\theta_{x t}^{k}\right|_{L^{2}}^{2}(s) d s \\
\leq & C c_{1}^{10} \int_{\tau}^{t}\left(1+\left|\theta_{x}^{k}\right|_{L^{2}}^{2}\right)\left(1+\left|\sqrt{\rho^{k}} \theta_{t}^{k}\right|_{L^{2}}^{2}\right)^{2}(s) d s \\
& +C c_{1}^{4} \int_{\tau}^{t}\left|u_{x t}^{k-1}\right|_{L^{2}}^{2}(s) d s+\left|\sqrt{\rho^{k} \varphi_{1}^{k}} \theta_{t}^{k}(\tau)\right|_{L^{2}}^{2},
\end{aligned}
$$

where $C$ is a positive constant.
We estimate $\left|\sqrt{\rho^{k} \varphi_{1}^{k}} \theta_{t}^{k}(\tau)\right|_{L^{2}}^{2}$ as follows: multiplying (17) by $\theta_{t}^{k}$ and integrating it over $(0,1)$, we obtain

$$
\begin{aligned}
& \int_{0}^{1} \rho^{k} \varphi_{1}^{k}\left|\theta_{t}^{k}\right|^{2} d x \leq \int_{0}^{1}\left(\varphi_{1}^{k}\right)^{-1}\left[\left(\rho^{k}\right)^{3}\left|\varphi_{2}^{k}\right|^{2}\left|u_{x}^{k-1}\right|^{2}\right. \\
& +\left(\rho^{k}\right)^{-1}\left|\psi^{k}\right|^{2}\left|u_{x}^{k-1}\right|^{2} \\
& \left.+\left(\rho^{k}\right)^{-1}\left|H_{p}^{\mu_{1}} \theta^{k}-\left(u_{x}^{k}\right)^{2}\right|^{2}\right] d x+\int_{0}^{1} \rho^{k}\left|\varphi_{1}^{k}\right| \\
& \cdot\left|u^{k-1}\right|^{2}\left|\theta_{x}^{k}\right|^{2} d x .
\end{aligned}
$$

Next, we get

$$
\begin{gathered}
\int_{0}^{1} \rho^{k} \varphi_{1}^{k}\left|\theta_{t}^{k}\right|^{2} d x \leq C \int_{0}^{1}\left(\rho^{k}\left|u^{k-1}\right|^{2}\left|\theta_{x}^{k}\right|^{2}\right. \\
+\left(\rho^{k}\right)^{3}\left|u_{x}^{k-1}\right|^{2}+\left(\rho^{k}\right)^{-1}\left|u_{x}^{k-1}\right|^{2} \\
\left.+\left(\rho^{k}\right)^{-1}\left|H_{p}^{\mu_{1}} \theta^{k}-\left(u_{x}^{k}\right)^{2}\right|^{2}\right) d x
\end{gathered}
$$

and, according to the smoothness of $\left(\rho^{k}, u^{k}, \theta^{k}\right)$, we have

$$
\begin{aligned}
& \lim _{\tau \rightarrow 0} \int_{0}^{1}\left(\rho^{k}\left|u^{k-1}\right|^{2}\left|\theta_{x}^{k}\right|^{2}+\left(\rho^{k}\right)^{3}\left|u_{x}^{k-1}\right|^{2}\right. \\
& +\left(\rho^{k}\right)^{-1}\left|u_{x}^{k-1}\right|^{2} \\
& \left.\quad+\left(\rho^{k}\right)^{-1}\left|H_{p}^{\mu_{1}} \theta^{k}-\left(u_{x}^{k}\right)^{2}\right|^{2}\right) d x \leq C c_{0}^{5},
\end{aligned}
$$

where $C$ is a positive constant.

We have

$$
\begin{aligned}
\frac{d}{d t}\left|\theta_{x}^{k}\right|_{L^{2}}^{2} & \leq 2 \int_{0}^{1} \theta_{x}^{k} \theta_{x t}^{k} d x \leq 2\left|\theta_{x}^{k}\right|_{L^{2}}\left|\theta_{x t}^{k}\right|_{L^{2}} \\
& \leq\left|\theta_{x}^{k}\right|_{L^{2}}^{2}+\left|\theta_{x t}^{k}\right|_{L^{2}}^{2}
\end{aligned}
$$

by Gronwall's inequality, it follows that

$$
\left|\theta_{x}^{k}\right|_{L^{2}}^{2} \leq \exp \{t\}\left(\left|\theta_{x}^{k}(0)\right|_{L^{2}}^{2}+\int_{0}^{t}\left|\theta_{x t}^{k}\right|_{L^{2}}^{2} d s\right)
$$

for $0<t<\min \left\{T_{1}, T_{2}\right\}$,

$$
\left|\theta_{x}^{k}\right|_{L^{2}}^{2} \leq C\left|\theta_{x}^{k}(0)\right|_{L^{2}}^{2}+C \int_{0}^{t}\left|\theta_{x t}^{k}\right|_{L^{2}}^{2} d s
$$

Due to (44), (47), and (50), we get

$$
\begin{aligned}
& \left|\theta_{x}^{k}\right|_{L^{2}}^{2}+\left|\sqrt{\rho^{k}} \theta_{t}^{k}(t)\right|_{L^{2}}^{2}+\int_{\tau}^{t}\left|\theta_{x t}^{k}\right|_{L^{2}}^{2}(s) d s \\
& \quad \leq C c_{1}^{10} \int_{\tau}^{t}\left(1+\left|\theta_{x}^{k}\right|_{L^{2}}^{2}\right)\left(1+\left|\sqrt{\rho^{k}} \theta_{t}^{k}\right|_{L^{2}}^{2}\right)^{2} d s+C c_{1}^{5} .
\end{aligned}
$$


Advances in Mathematical Physics

7

Denote $\Gamma(t)=1+\left|\theta_{x}^{k}\right|_{L^{2}}^{2}+\left|\sqrt{\rho^{k}} \theta_{t}^{k}\right|_{L^{2}}^{2}$; we get

$$
\Gamma(t) \leq C c_{1}^{10} \int_{0}^{t} \Gamma(t)^{3} d s+C c_{1}^{5}
$$

by Gronwall's inequality, $\exists T_{3} \leq T_{2}$ ( $T_{3}$ has nothing to do with $k$ ), it follows that

$$
\Gamma(t) \leq C c_{1}^{5}
$$

Note that, for a given function $\omega$, by a simple computatimon, we have

$$
\begin{aligned}
& \left.||\left(\left(\theta_{x}^{k}\right)^{2}+\mu_{1}\right)^{(p-2) / 2} \theta_{x}^{k}\right]_{t} \theta_{x t}^{k} \mid \\
& \quad=\left(\left(\theta_{x}^{k}\right)^{2}+\mu_{1}\right)^{(p-4) / 2}\left[(r-1)\left(\theta_{x}^{k}\right)^{2}+\mu_{1}\right]\left|\theta_{x t}^{k}\right|^{2} \\
& \quad \geq\left(\left(\theta_{x}^{k}\right)^{2}+\mu_{1}\right)^{(p-4) / 2}\left[\left(\theta_{x}^{k}\right)^{2}+\mu_{1}\right]\left|\theta_{x t}^{k}\right|^{2} \\
& \quad=\left(\left(\theta_{x}^{k}\right)^{2}+\mu_{1}\right)^{(p-2) / 2}\left|\theta_{x t}^{k}\right|^{2} \geq \mu_{1}^{(p-2) / 2}\left|\theta_{x t}^{k}\right|^{2} .
\end{aligned}
$$

Firstly, we need to do the following estimates: by (19)

$$
\begin{aligned}
& {\left[\left(\theta_{x}^{2}+\mu_{1}\right)^{(p-2) / 2} \theta_{x}\right]_{x}} \\
& =\rho^{k} \varphi_{1}^{k} \theta_{t}^{k}+\rho^{k} \varphi_{1}^{k} u^{k-1} \theta_{x}^{k}-\left(\rho^{k}\right)^{2} \varphi_{2}^{k} u_{x}^{k-1}+\psi^{k} u_{x}^{k-1} \\
& \quad-\left(u_{x}^{k-1}\right)^{2}
\end{aligned}
$$

and we have

$$
\begin{aligned}
& \left|\left[\left(\left(\theta_{x}^{k}\right)^{2}+\mu_{1}\right)^{(p-2) / 2} \theta_{x}^{k}\right]_{x}\right| \\
& \quad=\left(\left(\theta_{x}^{k}\right)^{2}+\mu_{1}\right)^{(p-4) / 2}\left[(p-1)\left(\theta_{x}^{k}\right)^{2}+\mu_{1}\right]\left|\theta_{x x}^{k}\right| \\
& \quad \geq\left(\left(\theta_{x}^{k}\right)^{2}+\mu_{1}\right)^{(p-4) / 2}\left[\left(\theta_{x}^{k}\right)^{2}+\mu_{1}\right]\left|\theta_{x x}^{k}\right| \\
& \quad=\left(\left(\theta_{x}^{k}\right)^{2}+\mu_{1}\right)^{(p-2) / 2}\left|\theta_{x x}^{k}\right| \geq \mu_{1}^{(p-2) / 2}\left|\theta_{x x}^{k}\right| .
\end{aligned}
$$

taking it by $L^{2}$-norm, we get

$$
\begin{aligned}
& \left|\theta_{x x}^{k}\right|_{L^{2}} \leq \mu_{1}^{(2-p) / 2}\left[\left|\rho^{k} \varphi_{1}^{k} \theta_{t}^{k}\right|_{L^{2}}+\left|\rho^{k} \varphi_{1}^{k} u^{k-1} \theta_{x}^{k}\right|_{L^{2}}\right. \\
& \left.+\left|\left(\rho^{k}\right)^{2} \varphi_{2}^{k} u_{x}^{k-1}\right|_{L^{2}}+\left|\psi^{k} u_{x}^{k-1}\right|_{L^{2}}+\left|\left(u_{x}^{k-1}\right)^{2}\right|_{L^{2}}\right] \\
& \leq C\left[\left|\rho^{k}\right|_{L^{\infty}}^{1 / 2}\left|\sqrt{\rho^{k}} \theta_{t}^{k}\right|_{L^{2}}+\left|\rho^{k}\right|_{L^{\infty}}\left|u^{k-1}\right|_{L^{\infty}}\left|\theta_{x}^{k}\right|_{L^{2}}\right. \\
& \left.+\left|\rho^{k}\right|_{L^{\infty}}^{2}\left|u_{x}^{k-1}\right|_{L^{2}}+\left|u_{x}^{k-1}\right|_{L^{2}}+\left|u_{x}^{k-1}\right|_{L^{\infty}}\left|u_{x}^{k-1}\right|_{L^{2}}\right]
\end{aligned}
$$

$$
\begin{aligned}
& \leq C\left[\left|\rho_{x}^{k}\right|_{L^{2}}^{1 / 2}\left|\sqrt{\rho^{k}} \theta_{t}^{k}\right|_{L^{2}}+\left|\rho_{x}^{k}\right|_{L^{2}}\left|u_{x}^{k-1}\right|_{L^{2}}\left|\theta_{x}^{k}\right|_{L^{2}}\right. \\
& \left.+\left|\rho_{x}^{k}\right|_{L^{2}}^{2}\left|u_{x}^{k-1}\right|_{L^{2}}+\left|u_{x}^{k-1}\right|_{L^{2}}+\left|u_{x x}^{k-1}\right|_{L^{2}}\left|u_{x}^{k-1}\right|_{L^{2}}\right] \\
& \leq C c_{1}^{4} c_{2} .
\end{aligned}
$$

(iii) Estimation for $u^{k}(t)$.

Differentiating (20) with respect to $t$, multiplying it by $u_{t}^{k}$, and integrating it over $(0,1)$ on $x$, we derive

$$
\begin{aligned}
& \frac{1}{2} \frac{d}{d t} \int_{0}^{1} \rho^{k}\left|u_{t}^{k}\right|^{2} d x \\
& \quad+\int_{0}^{1}\left[\left(u_{x}^{2}+\mu_{2}\right)^{(q-2) / 2} u_{x}\right]_{t} u_{x t}^{k} d x \\
& \quad=\frac{1}{2} \int_{0}^{1} \rho_{t}^{k}\left|u_{t}^{k}\right|^{2} d x \\
& \quad-\int_{0}^{1}\left[\rho_{t}^{k} u^{k-1} u_{x}^{k}+\rho^{k} u_{t}^{k-1} u_{x}^{k}+\rho^{k} u^{k-1} u_{x t}^{k}\right] u_{t}^{k} d x \\
& \quad+\int_{0}^{1} \psi_{t}^{k} u_{x t}^{k} d x=\sum_{j=1}^{5} I_{j} .
\end{aligned}
$$

Then, using the Sobolev inequality, Young's inequality, Lemma 3, and (25), (30), and (31), we obtain

$$
\begin{aligned}
I_{1} & =\frac{1}{2} \int_{0}^{1} \rho_{t}^{k}\left|u_{t}^{k}\right|^{2} d x \leq \int_{0}^{1} \rho^{k}\left|u^{k-1}\right|\left|u_{t}^{k}\right|\left|u_{x t}^{k}\right| d x \\
& \leq\left|\rho^{k}\right|_{L^{\infty}}^{1 / 2}\left|u^{k-1}\right|_{L^{\infty}}\left|\sqrt{\rho^{k}} u_{t}^{k}\right|_{L^{2}}\left|u_{x t}^{k}\right|_{L^{2}} \\
& \leq \eta_{1}\left|u_{x t}^{k}\right|_{L^{2}}^{2}+C_{\eta_{1}} c_{1}^{3}\left|\sqrt{\rho^{k}} u_{t}^{k}\right|_{L^{2}}^{2}, \\
I_{2} & =-\int_{0}^{1} \rho_{t}^{k} u^{k-1} u_{x}^{k} u_{t}^{k} d x \leq \int_{0}^{1}\left|\rho_{t}^{k}\right|\left|u^{k-1}\right|\left|u_{x}^{k}\right|\left|u_{t}^{k}\right| d x \\
& \leq\left|\rho_{t}^{k}\right|_{L^{2}}\left|u^{k-1}\right|_{L^{\infty}}^{2}\left|u_{x}^{k}\right|_{L^{2}}\left|u_{t}^{k}\right|_{L^{\infty}} \\
& \leq \eta_{1}\left|u_{x t}^{k}\right|_{L^{2}}^{2}+C_{\eta_{1}} c_{1}^{6}\left|u_{x}^{k}\right|_{L^{2}}^{2}, \\
I_{3} & =-\int_{0}^{1} \rho^{k} u_{t}^{k-1} u_{x}^{k} u_{t}^{k} d x \leq \int_{0}^{1} \rho^{k}\left|u_{t}^{k-1}\right|\left|u_{x}^{k}\right|\left|u_{t}^{k}\right| d x \\
& \leq\left|\rho^{k}\right|_{L^{\infty}}^{1 / 2}\left|u_{t}^{k-1}\right|_{L^{\infty}}\left|u_{x}^{k}\right|_{L^{2}}\left|\sqrt{\rho^{k}} u_{t}^{k}\right|_{L^{2}} \\
& \leq c_{0}^{1 / 2}\left|u_{x t}^{k-1}\right| u_{L^{2}}\left|u_{x}^{k}\right|_{L^{2}}\left|\sqrt{\rho^{k}} u_{t}^{k}\right|_{L^{2}} \\
L_{L^{2}} & \left.\sqrt{\rho^{k}} u_{t}^{k}\right|_{L^{2}} ^{2}+\frac{1}{4} c_{0} c_{1}^{-1}\left|u_{x}^{k}\right|_{L^{2}}^{2},
\end{aligned}
$$


8

Advances in Mathematical Physics

$$
\begin{aligned}
I_{4} & =-\int_{0}^{1} \rho^{k} u^{k-1} u_{x t}^{k} u_{t}^{k} d x \leq \int_{0}^{1} \rho^{k}\left|u^{k-1}\right|\left|u_{t}^{k}\right|\left|u_{x t}^{k}\right| d x \\
& \leq\left|\rho^{k}\right|_{L^{\infty}}^{1 / 2}\left|u^{k-1}\right|_{L^{\infty}}\left|\sqrt{\rho^{k} u_{t}^{k}}\right|_{L^{2}}\left|u_{x t}^{k}\right|_{L^{2}} \\
& \leq \eta_{1}\left|u_{x t}^{k}\right|_{L^{2}}^{2}+C_{\eta_{1}} c_{1}^{3}\left|\sqrt{\rho^{k}} u_{t}^{k}\right|_{L^{2}}^{2}, \\
I_{5} & =\int_{0}^{1}\left|\psi_{t}^{k}\right|\left|u_{x t}^{k}\right| d x \\
& =\int_{0}^{1}\left|\psi_{1}^{k}\right|\left|\theta_{t}^{k}\right|\left|u_{x t}^{k}\right| d x+\int_{0}^{1}\left|\psi_{2}^{k}\right|\left|\rho_{t}^{k}\right|\left|u_{x t}^{k}\right| d x \\
& \leq C\left|\sqrt{\rho^{k}} \theta_{t}^{k}\right|_{L^{2}}\left|u_{x t}^{k}\right|_{L^{2}}+C\left|\rho_{t}^{k}\right|_{L^{2}}\left|u_{x t}^{k}\right|_{L^{2}} \\
& \leq 2 \eta_{1}\left|u_{x t}^{k}\right|_{L^{2}}^{2}+C_{\eta_{1}} c_{1}^{4}+C_{\eta_{1}} c_{1}^{5} .
\end{aligned}
$$

Substituting $I_{j}(j=1,2, \ldots, 5)$ into (33), after choosing $\eta=\mu_{2}^{(q-2) / 2} / 10$

$$
\begin{aligned}
\frac{1}{2} \frac{d}{d t} \mid & \left.\sqrt{\rho^{k}} u_{t}^{k}(t)\right|_{L^{2}} ^{2}+\frac{1}{2} \mu_{2}^{(q-2) / 2}\left|u_{x t}^{k}\right|_{L^{2}}^{2} \\
\leq & C c_{1}^{3}\left|\sqrt{\rho^{k}} u_{t}^{k}\right|_{L^{2}}^{2}+C c_{1}^{6}\left|u_{x}^{k}\right|_{L^{2}}^{2} \\
& +c_{1}^{-1}\left|u_{x t}^{k-1}\right|_{L^{2}}\left|\sqrt{\rho^{k}} u_{t}^{k}\right|_{L^{2}}+\frac{1}{4} c_{0} c_{1}^{-1}\left|u_{x}^{k}\right|_{L^{2}}+C c_{1}^{5}
\end{aligned}
$$

We estimate $\left|\sqrt{\rho^{k}} u_{t}^{k}(\tau)\right|_{L^{2}}^{2}$ as follows: multiplying (20) by $u_{t}^{k}$ and integrating it over $(0,1)$, we obtain

$$
\begin{aligned}
& \int_{0}^{1} \rho^{k}\left|u_{t}^{k}\right|^{2} d x \\
& \leq 2 \int_{0}^{1}\left(\rho^{k}\left|u^{k-1}\right|^{2}\left|u_{x}^{k}\right|^{2}+\left(\rho^{k}\right)^{-1}\left|H_{q}^{\mu_{2}} u^{k}-\psi_{x}\right|^{2}\right) d x .
\end{aligned}
$$

According to the smoothness of $\left(\rho^{k}, u^{k}, \theta^{k}\right)$, we have

$$
\begin{aligned}
& \lim _{\tau \rightarrow 0} \int_{0}^{1}\left(\rho^{k}\left|u^{k-1}\right|^{2}\left|u_{x}^{k}\right|^{2}+\left(\rho^{k}\right)^{-1}\left|L_{p} u^{k}+\psi_{x}\right|^{2}\right) d x \\
& \quad \leq\left|\rho_{0}\right|_{L^{\infty}}\left|u_{0}\right|_{L^{\infty}}^{2}\left|u_{0 x}\right|_{L^{2}}^{2}+|g|_{L^{2}}^{2} \leq C c_{0}^{5},
\end{aligned}
$$

where $C$ is a positive constant.

In the same way,

$$
\left|u_{x}^{k}\right|_{L^{2}}^{2} \leq C\left|u_{x}^{k}(0)\right|_{L^{2}}^{2}+C \int_{0}^{t}\left|u_{x t}^{k}\right|_{L^{2}}^{2} d s
$$

Integrating $(60)$ over $(0, t) \subset(0, T)$, combining with (62) and (63) we have

$$
\begin{aligned}
& \left|u_{x}^{k}\right|_{L^{2}}^{2}+\left|\sqrt{\rho^{k}} u_{t}^{k}(t)\right|_{L^{2}}^{2}+\int_{0}^{t}\left|u_{x t}^{k}\right|_{L^{2}}^{2} \\
& \quad \leq C \int_{0}^{t}\left(c_{1}^{6}+c_{1}^{-1}\left|u_{x t}^{k-1}\right|_{L^{2}}\right) \\
& \cdot\left(\left|\sqrt{\rho^{k}} u_{t}^{k}(t)\right|_{L^{2}}^{2}+\left|u_{x}^{k}\right|_{L^{2}}^{2}\right) d s+C c_{1}^{5} t+C c_{0}^{5},
\end{aligned}
$$

and for $0<t<\min \left\{T_{1}, T_{4}\right\}, T_{4} \leq c_{0}^{5} / c_{1}^{5}$, we get

$$
\begin{aligned}
& \left|u_{x}^{k}\right|_{L^{2}}^{2}+\left|\sqrt{\rho^{k}} u_{t}^{k}(t)\right|_{L^{2}}^{2}+\int_{0}^{t}\left|u_{x t}^{k}\right|_{L^{2}}^{2} \\
& \leq C \int_{0}^{t}\left(c_{1}^{6}+c_{1}^{-1}\left|u_{x t}^{k-1}\right|_{L^{2}}\right) \\
& \cdot\left(\left|\sqrt{\rho^{k}} u_{t}^{k}(t)\right|_{L^{2}}^{2}+\left|u_{x}^{k}\right|_{L^{2}}^{2}\right) d s+C c_{0}^{5},
\end{aligned}
$$

and, by Gronwall's inequality, $\exists T_{5} \leq T_{4}$ ( $T_{5}$ has nothing to do with $k$ ), it follows that

$$
\left|u_{x}^{k}\right|_{L^{2}}^{2}+\left|\sqrt{\rho^{k}} u_{t}^{k}(t)\right|_{L^{2}}^{2}+\int_{0}^{t}\left|u_{x t}^{k}\right|_{L^{2}}^{2}(s) d s \leq C c_{0}^{5}=c_{1} .
$$

Secondly, we need to do the following estimates. By (20)

$$
\begin{aligned}
& {\left[\left(u_{x}^{2}+\mu_{2}\right)^{(q-2) / 2} u_{x}\right]_{x}} \\
& \quad=\rho^{k} u_{t}^{k}+\rho^{k} u^{k-1} u_{x}^{k-1}+\phi_{1}^{k-1} \theta_{x}^{k-1}+\phi_{2}^{k-1} \rho_{x}^{k},
\end{aligned}
$$

we have

$$
\begin{aligned}
& \left.||\left(\left(u_{x}^{k}\right)^{2}+\mu_{2}\right)^{(q-2) / 2} u_{x}^{k}\right]_{x} \mid \\
& \quad=\left(\left(u_{x}^{k}\right)^{2}+\mu_{2}\right)^{(q-4) / 2}\left[(q-1)\left(u_{x}^{k}\right)^{2}+\mu_{2}\right]\left|u_{x x}^{k}\right| \\
& \quad \geq\left(\left(u_{x}^{k}\right)^{2}+\mu_{2}\right)^{(q-4) / 2}\left[\left(u_{x}^{k}\right)^{2}+\mu_{2}\right]\left|u_{x x}^{k}\right| \\
& \quad=\left(\left(u_{x}^{k}\right)^{2}+\mu_{2}\right)^{(q-2) / 2}\left|u_{x x}^{k}\right| \geq \mu_{2}^{(q-2) / 2}\left|u_{x x}^{k}\right|,
\end{aligned}
$$

and, taking it by $L^{2}$-norm, using Lemma 3 , we get

$$
\begin{aligned}
& \left|u_{x x}^{k}\right|_{L^{2}} \leq \mu_{2}^{(2-q) / 2}\left[\left|\rho^{k} u_{t}^{k}\right|_{L^{2}}+\left|\rho^{k} u^{k-1} u_{x}^{k}\right|_{L^{2}}+\left|\psi_{1}^{k} \theta_{x}^{k}\right|_{L^{2}}\right. \\
& \left.\quad+\left|\psi_{2}^{k} \rho_{x}^{k}\right|_{L^{2}}\right] \leq C\left[\left|\rho^{k}\right|_{L^{\infty}}^{1 / 2}\left|\sqrt{\rho^{k} u_{t}^{k}}\right|_{L^{2}}\right. \\
& \left.\quad+\left|\rho^{k}\right|_{L^{\infty}}\left|u^{k-1}\right|_{L^{\infty}}\left|u_{x}^{k}\right|_{L^{q}}+\left|\theta_{x}^{k}\right|_{L^{2}}+\left|\rho_{x}^{k}\right|_{L^{2}}\right] \\
& \quad \leq C\left[\left|\rho_{x}^{k}\right|_{L^{2}}^{1 / 2}\left|\sqrt{\rho^{k}} u_{t}^{k}\right|_{L^{2}}+\left|\rho_{x}^{k}\right|_{L^{2}}\left|u_{x}^{k-1}\right|_{L^{9}}\left|u_{x}^{k}\right|_{L^{q}}\right. \\
& \left.\quad+\left|\theta_{x}^{k}\right|_{L^{2}}+\left|\rho_{x}^{k}\right|_{L^{2}}\right] \leq C c_{1}^{3}=c_{2}=C c_{0}^{15},
\end{aligned}
$$


Advances in Mathematical Physics

9

where $C$ is a positive constant. For $T_{6}=\min \left[T_{1}, T_{2}, T_{3}\right.$, $\left.T_{4}, T_{5}\right]$, we get

$$
\begin{aligned}
& \sup _{0 \leq s \leq T_{6}}\left|u_{x}^{k}\right|_{L^{2}}(s)+\int_{0}^{T_{6}}\left|u_{x t}^{k}\right|_{L^{2}}^{2}(s) d s \leq c_{1}, \\
& \sup _{0 \leq s \leq T_{6}}\left|u_{x x}^{k}\right|_{L^{2}}(s) \leq c_{2}, \\
& \sup _{0 \leq s \leq T_{6}}\left|\theta_{x}^{k}\right|_{L^{2}}^{2}(s)+\left|\sqrt{\rho^{k}} \theta_{t}^{k}(t)\right|_{L^{2}}^{2}(s)+\int_{0}^{T_{6}}\left|\theta_{x t}^{k}\right|_{L^{2}}^{2}(s) d s \\
& \leq C c_{1}^{5}, \\
& \sup _{0 \leq s \leq T_{6}}\left|u_{x}^{k}\right|_{L^{2}}^{2}(s)+\left|\sqrt{\rho^{k}} u_{t}^{k}(t)\right|_{L^{2}}^{2}(s)+\int_{0}^{T_{6}}\left|u_{x t}^{k}\right|_{L^{2}}^{2}(s) d s \\
& \quad \leq c_{1}, \\
& \sup _{0 \leq s \leq T_{6}}\left|\theta_{x x}^{k}\right|_{L^{2}}(s)<C c_{1}^{4} c_{2}, \\
& \sup _{0 \leq s \leq T_{6}}\left|\rho^{k}(t)\right|_{H^{1}}(s) \leq C c_{0}, \\
& \sup _{0 \leq s \leq T_{6}}\left|\rho_{t}^{k}(t)\right|_{L^{2}}(s) \leq C c_{1}^{2} .
\end{aligned}
$$

3.2. Convergence. In this subsection, the approximate soldtons $\left(\rho^{k}, u^{k}, \theta^{k}\right)$ strongly converging to the solution of (3)-(4) will be proved. To this end, we define

$$
\begin{aligned}
& \bar{\rho}^{k+1}=\rho^{k+1}-\rho^{k}, \\
& \bar{u}^{k+1}=u^{k+1}-u^{k}, \\
& \bar{\theta}^{k+1}=\theta^{k+1}-\theta^{k},
\end{aligned}
$$

and then we can easily verify that $\bar{\rho}^{k+1}, \bar{u}^{k+1}, \bar{\theta}^{k+1}$ satisfy the system of equations

$$
\begin{aligned}
& \bar{\rho}_{t}^{k+1}+\left(\bar{\rho}^{k+1} u^{k}\right)_{x}+\left(\rho^{k} \bar{u}^{k}\right)_{x}=0, \\
& \rho^{k+1} \varphi_{1}^{k+1} \theta_{t}^{k+1}+\rho^{k+1} \varphi_{1}^{k+1} u^{k} \theta_{x}^{k+1}-\left(\rho^{k+1}\right)^{2} \varphi_{2}^{k+1} u_{x}^{k} \\
& \quad+H_{p}^{\mu_{1}} \theta^{k+1}+\psi^{k+1} u_{x}^{k}-\left(u_{x}^{k}\right)^{2}-\left[\rho^{k} \varphi_{1}^{k} \theta_{t}^{k}\right. \\
& +\rho^{k} \varphi_{1}^{k} u^{k-1} \theta_{x}^{k}-\left(\rho^{k}\right)^{2} \varphi_{2}^{k} u_{x}^{k-1}+H_{p}^{\mu_{1}} \theta^{k}+\psi^{k} u_{x}^{k-1} \\
& \left.\quad-\left(u_{x}^{k-1}\right)^{2}\right]=0, \quad(x, t) \in \Omega_{T}, \\
& \rho^{k+1} \bar{u}_{t}^{k+1}+\rho^{k+1} u^{k} \bar{u}_{x}^{k+1}+\left(H_{q}^{\mu_{2}} u^{k+1}-H_{q}^{\mu_{2}} u^{k}\right) \\
& +\left(\psi^{k+1}\right)_{x}-\left(\psi^{k}\right)_{x}=\bar{\rho}^{k+1}\left(-u_{t}^{k}-u^{k-1} u_{x}^{k}\right) \\
& \quad-\rho^{k+1} \bar{u}^{k} u_{x}^{k},
\end{aligned}
$$

with the initial and boundary conditions

$$
\begin{aligned}
\bar{u}^{k+1} & =0, \\
\bar{\theta}^{k+1} & =0, \\
\quad \text { on } \partial \Omega \times(0, T), & \\
\bar{\rho}^{k+1}(x, 0) & =0, \\
\bar{u}^{k+1}(x, 0) & =0, \\
\bar{\theta}^{k+1}(x, 0) & =0,
\end{aligned}
$$

in $\Omega$.

Multiplying (72) by $\bar{\rho}^{k+1}$ and integrating it over $(0,1)$, we obtain

$$
\begin{aligned}
\frac{d}{d t}\left|\bar{\rho}^{k+1}\right|_{L^{2}}^{2} & \leq C\left|\bar{\rho}^{k+1}\right|_{L^{2}}^{2}\left|u_{x}^{k}\right|_{L^{\infty}}+\left|\rho^{k}\right|_{H^{1}}\left|\bar{u}_{x}^{k}\right|_{L^{2}}\left|\bar{\rho}^{k+1}\right|_{L^{2}} \\
& \leq B_{\eta}^{k}(t)\left|\bar{\rho}^{k+1}\right|_{L^{2}}^{2}+\eta\left|\bar{u}_{x}^{k}\right|_{L^{2}}^{2}
\end{aligned}
$$

where $B_{\eta}^{k}(t)=C\left|u_{x x}^{k}\right|_{L^{2}}+C_{\eta}\left|\rho^{k}\right|_{H^{1}}^{2}$, for all $t<T_{1}$ and $k \geq 1$.

Multiplying (73) by $\bar{\theta}^{k+1}$ and integrating it over $(0,1)$, we obtain

$$
\begin{aligned}
& \frac{1}{2} \frac{d}{d t} \int_{0}^{1} \rho^{k+1} \varphi_{1}^{k+1}\left|\bar{\theta}^{k+1}\right|^{2} d x \\
& \quad+\int_{0}^{1}\left(H_{p}^{\mu_{1}} \theta^{k+1}-H_{p}^{\mu_{1}} \theta^{k}\right) \bar{\theta}^{k+1} d x \\
& \quad=\frac{1}{2} \int_{0}^{1}\left[\rho^{k+1} \varphi_{1}^{k+1}\right]_{t}\left|\bar{\theta}^{k+1}\right|^{2} d x \\
& \quad-\int_{0}^{1} \rho^{k+1} \varphi_{1}^{k+1} \theta_{t}^{k} \bar{\theta}^{k+1} d x+\int_{0}^{1} \rho^{k} \varphi_{1}^{k} \theta_{t}^{k} \bar{\theta}^{k+1} d x \\
& \quad-\int_{0}^{1}\left[\rho^{k+1} \varphi_{1}^{k+1} u^{k} \theta_{x}^{k+1}-\rho^{k} \varphi_{1}^{k} u^{k-1} \theta_{x}^{k}\right] \bar{\theta}^{k+1} d x \\
& \quad-\int_{0}^{1}\left[\psi^{k+1} u_{x}^{k}-\psi^{k} u_{x}^{k-1}\right] \bar{\theta}^{k+1} d x \\
& \quad+\int_{0}^{1}\left[\left(u_{x}^{k}\right)^{2}-\left(u_{x}^{k-1}\right)^{2}\right] \bar{\theta}^{k+1} d x \\
& \quad-\int_{0}^{1}\left[\left(\rho^{k+1}\right)^{2} \varphi_{2}^{k+1} u_{x}^{k}-\left(\rho^{k}\right)^{2} \varphi_{2}^{k} u_{x}^{k-1}\right] \bar{\theta}^{k+1} d x \\
& \quad=\sum_{j=1}^{6} I_{j} \cdot
\end{aligned}
$$


Using the Sobolev inequality, Young's inequality, and Lemma 3, we obtain

$$
\begin{aligned}
I_{1}= & \frac{1}{2} \int_{0}^{1}\left[\rho^{k+1} \varphi_{1}^{k+1}\right]_{t}\left|\bar{\theta}^{k+1}\right|^{2} d x \\
= & \frac{1}{2} \int_{0}^{1} \rho_{t}^{k+1} \varphi_{1}^{k+1}\left|\bar{\theta}^{k+1}\right|^{2} d x \\
& +\frac{1}{2} \int_{0}^{1} \rho^{k+1} \varphi_{11}^{k+1} \theta_{t}^{k+1}\left|\bar{\theta}^{k+1}\right|^{2} d x \\
& +\frac{1}{2} \int_{0}^{1} \rho^{k+1} \varphi_{12}^{k+1} \rho_{t}^{k+1}\left|\bar{\theta}^{k+1}\right|^{2} d x=\sum_{j=1}^{3} I_{1 j} .
\end{aligned}
$$

Here,

$$
\begin{aligned}
I_{11} & =\frac{1}{2} \int_{0}^{1} \rho_{t}^{k+1} \varphi_{1}^{k+1}\left|\bar{\theta}^{k+1}\right|^{2} d x \\
& \leq\left. C\left|\int_{0}^{1} \rho_{t}^{k+1}\right| \bar{\theta}^{k+1}\right|^{2} d x \mid \\
& \leq C\left|\int_{0}^{1} \rho^{k+1} u^{k} \bar{\theta}^{k+1} \bar{\theta}_{x}^{k+1} d x\right| \\
& \leq C\left|\rho^{k+1}\right|_{L^{\infty}}^{1 / 2}\left|\sqrt{\rho^{k+1}} \bar{\theta}^{k+1}\right|_{L^{2}}\left|u^{k}\right|_{L^{\infty}}\left|\bar{\theta}_{x}^{k+1}\right| \\
& \leq \eta\left|\bar{\theta}_{x}^{k+1}\right|_{L^{2}}^{2}+C_{\eta}\left|\rho_{x}^{k+1}\right|_{L^{2}}\left|u_{x}^{k}\right|_{L^{2}}^{2}\left|\sqrt{\rho^{k+1}} \bar{\theta}^{k+1}\right|_{L^{2}}^{2} \\
& \leq \eta\left|\bar{\theta}_{x}^{k+1}\right|_{L^{2}}^{2}+C_{\eta}\left|\rho_{x}^{k+1}\right|_{L^{2}}^{3}\left|u_{x}^{k}\right|_{L^{2}}^{2}\left|\sqrt{\rho^{k+1}} \bar{\theta}^{k+1}\right|_{L^{2}}^{2} \\
& \leq \frac{1}{2} \int_{0}^{1} \rho^{k+1} \varphi_{11}^{k+1} \theta_{t}^{k+1}\left|\bar{\theta}^{k+1}\right|^{2} d x \\
& \leq\left. C\left|\int_{0}^{1} \rho^{k+1} \theta_{t}^{k+1}\right| \bar{\theta}^{k+1}\right|^{2} d x \mid \\
& \leq C\left|\theta_{t}^{k+1}\right|_{L^{\infty}}^{k+1}\left|\sqrt{\rho^{k+1}} \bar{\theta}^{k+1}\right|_{L^{2}}^{2}\left|\sqrt{\rho^{k+1}} \bar{\theta}^{k+1}\right|_{L^{2}}^{2}, \rho_{t}^{k+1}\left|\bar{\theta}^{k+1}\right|^{2} d x \mid
\end{aligned}
$$

In the same way, we have

$$
\begin{aligned}
I_{2}= & -\int_{0}^{1} \rho^{k+1} \varphi_{1}^{k+1} \theta_{t}^{k} \bar{\theta}^{k+1} d x+\int_{0}^{1} \rho^{k} \varphi_{1}^{k} \theta_{t}^{k} \bar{\theta}^{k+1} d x \\
= & -\int_{0}^{1} \bar{\rho}^{k+1} \varphi_{1}^{k+1} \theta_{t}^{k} \bar{\theta}^{k+1} d x+\int_{0}^{1} \rho^{k} \bar{\varphi}_{1}^{k+1} \theta_{t}^{k} \bar{\theta}^{k+1} d x \\
\leq & C\left|\int_{0}^{1} \bar{\rho}^{k+1} \theta_{t}^{k} \bar{\theta}^{k+1} d x\right|+C\left|\int_{0}^{1} \rho^{k-1} \bar{\rho}^{k+1} \theta_{t}^{k} \bar{\theta}^{k+1} d x\right| \\
& +C\left|\int_{0}^{1} \rho^{k} \bar{\theta}^{k+1} \theta_{t}^{k} \bar{\theta}^{k+1} d x\right|=\sum_{j=1}^{3} I_{2 j} .
\end{aligned}
$$

Here,

$$
\begin{aligned}
I_{21} & =C\left|\int_{0}^{1} \bar{\rho}^{k+1} \theta_{t}^{k} \bar{\theta}^{k+1} d x\right| \leq C\left|\bar{\rho}^{k+1}\right|_{L^{2}}\left|\theta_{x t}^{k}\right|_{L^{2}}\left|\bar{\theta}_{x}^{k+1}\right|_{L^{2}} \\
& \leq \eta\left|\bar{\theta}_{x}^{k+1}\right|_{L^{2}}^{2}+C_{\eta}\left|\theta_{x t}^{k}\right|_{L^{2}}^{2}\left|\bar{\rho}^{k+1}\right|_{L^{2}}^{2}, \\
I_{22} & =C\left|\int_{0}^{1} \rho^{k} \bar{\rho}^{k+1} \theta_{t}^{k} \bar{\theta}^{k+1} d x\right| \\
& \leq C\left|\rho_{x}^{k}\right|_{L^{2}}\left|\bar{\rho}^{k+1}\right|{ }_{L^{2}}\left|\theta_{x t}^{k}\right|_{L^{2}}\left|\bar{\theta}_{x}^{k+1}\right|_{L^{2}} \\
& \leq \eta\left|\theta_{x}^{k+1}\right|_{L^{2}}^{2}+C_{\eta}\left|\rho_{x}^{k}\right|_{L^{2}}^{2}\left|\theta_{x t}^{k}\right|_{L^{2}}^{2}\left|\bar{\rho}^{k+1}\right|_{L^{2}}^{2}, \\
I_{23} & =C\left|\int_{0}^{1} \rho^{k} \bar{\theta}^{k+1} \theta_{t}^{k} \bar{\theta}^{k+1} d x\right| \\
& \leq C\left|\rho_{x}^{k}\right|_{L^{2}}\left|\theta_{x t}^{k}\right|_{L^{2}}\left|\sqrt{\rho^{k+1}} \bar{\theta}^{k+1}\right|_{L^{2}}^{2} .
\end{aligned}
$$

Following the same method, we obtain

$$
\begin{aligned}
& I_{3}=-\int_{0}^{1}\left[\rho^{k+1} \varphi_{1}^{k+1} u^{k} \theta_{x}^{k+1}-\rho^{k} \varphi_{1}^{k} u^{k-1} \theta_{x}^{k}\right] \bar{\theta}^{k+1} d x \\
& =-\int_{0}^{1} \bar{\rho}^{k+1} \varphi_{1}^{k+1} u^{k} \theta_{x}^{k+1} \bar{\theta}^{k+1} d x \\
& -\int_{0}^{1} \rho^{k} \bar{\varphi}_{1}^{k+1} u^{k} \theta_{x}^{k+1} \bar{\theta}^{k+1} d x \\
& -\int_{0}^{1} \rho^{k} \varphi_{1}^{k} \bar{u}^{k} \theta_{x}^{k+1} \bar{\theta}^{k+1} d x \\
& -\int_{0}^{1} \rho^{k} \varphi_{1}^{k} u^{k-1} \bar{\theta}_{x}^{k+1} \bar{\theta}^{k+1} d x=\sum_{j=1}^{4} I_{3 j}
\end{aligned}
$$

Here,

$$
\begin{aligned}
I_{31} & =-\int_{0}^{1} \bar{\rho}^{k+1} \varphi_{1}^{k+1} u^{k} \theta_{x}^{k+1} \bar{\theta}^{k+1} d x \\
& \leq C\left|\bar{\rho}^{k+1}\right|_{L^{2}}\left|u_{x}^{k}\right|_{L^{2}}\left|\theta_{x t}^{k+1}\right|_{L^{2}}\left|\bar{\theta}_{x}^{k+1}\right|_{L^{2}} \\
& \leq \eta\left|\bar{\theta}_{x}^{k+1}\right|_{L^{2}}^{2}+C_{\eta}\left|u_{x}^{k}\right|_{L^{2}}^{2}\left|\theta_{x t}^{k+1}\right|_{L^{2}}^{2}\left|\bar{\rho}^{k+1}\right|_{L^{2}}^{2},
\end{aligned}
$$


Advances in Mathematical Physics

11

$$
\begin{aligned}
I_{32}= & -\int_{0}^{1} \rho^{k} \bar{\varphi}_{1}^{k+1} u^{k} \theta_{x}^{k+1} \bar{\theta}^{k+1} d x \\
\leq & C\left|\int_{0}^{1} \rho^{k} \bar{\rho}^{k+1} u^{k} \theta_{x}^{k+1} \bar{\theta}^{k+1} d x\right| \\
& +C\left|\int_{0}^{1} \rho^{k} \bar{\theta}{ }^{k+1} u^{k} \theta_{x}^{k+1} \bar{\theta}^{k+1} d x\right| \\
\leq & \eta\left|\bar{\theta}_{x}^{k+1}\right|_{L^{2}}^{2}+C_{\eta}\left|u_{x}^{k}\right|_{L^{2}}^{2}\left|\rho_{x}^{k}\right|_{L^{2}}^{2}\left|\theta_{x t}^{k+1}\right|_{L^{2}}^{2}\left|\bar{\rho}^{k+1}\right|_{L^{2}}^{2} \\
& +C\left|u_{x}^{k}\right|_{L^{2}}\left|\rho_{x}^{k}\right|_{L^{2}}\left|\theta_{x t}^{k+1}\right|_{L^{2}}\left|\sqrt{\rho^{k+1}} \bar{\theta}^{k+1}\right|_{L^{2}}^{2}, \\
I_{33}= & \int_{0}^{1} \rho^{k} \varphi_{1}^{k} \bar{u}^{k} \theta_{x}^{k+1} \bar{\theta}^{k+1} d x \\
\leq & C\left|\bar{u}_{x}^{k}\right|_{L^{2}}\left|\rho_{x}^{k}\right|_{L^{2}}\left|\theta_{x t}^{k+1}\right|_{L^{2}}\left|\sqrt{\rho^{k+1}} \bar{\theta}^{k+1}\right|_{L^{2}} \\
\leq & C\left|\bar{u}_{x}^{k}\right|_{L^{2}}^{2}+C\left|\rho_{x}^{k}\right|_{L^{2}}^{2}\left|\theta_{x t}^{k+1}\right|_{L^{2}}^{2}\left|\sqrt{\rho^{k+1}} \bar{\theta}^{k+1}\right|_{L^{2}}^{2}, \\
I_{34}= & \int_{0}^{1} \rho^{k} \varphi_{1}^{k} u^{k-1} \theta_{x}^{k+1} \bar{\theta}^{k+1} d x \\
\leq & \left.C\left|\rho^{k}\right|_{L^{\infty}}\left|\sqrt{\rho^{k+1}} \bar{\theta}^{k+1}\right|\right|_{L^{2}}\left|u^{k-1}\right|_{L^{\infty}}\left|\bar{\theta}_{x}^{k+1}\right|_{L^{2}}^{2} \\
\leq & \eta\left|\bar{\theta}_{x}^{k}\right|_{L^{2}}^{2}+C_{\eta}\left|\rho_{x}^{k}\right|_{L^{2}}^{2}\left|u_{x}^{k-1}\right|_{L^{2}}^{2}\left|\sqrt{\rho^{k+1}} \bar{\theta}^{k+1}\right|_{L^{2}}^{2}
\end{aligned}
$$

In a similar way, we have

$$
\begin{aligned}
I_{4} & =-\int_{0}^{1}\left[\psi^{k+1} u_{x}^{k}-\psi^{k} u_{x}^{k-1}\right] \bar{\theta}^{k+1} d x \\
& =-\int_{0}^{1} \bar{\psi}^{k+1} u_{x}^{k} \bar{\theta}^{k+1} d x-\int_{0}^{1} \psi^{k} \bar{u}_{x}^{k} \bar{\theta}^{k+1} d x=\sum_{j=1}^{2} I_{4 j} .
\end{aligned}
$$

Here,

$$
\begin{aligned}
I_{41}= & -\int_{0}^{1} \bar{\psi}^{k+1} u_{x}^{k} \bar{\theta}^{k+1} d x \\
\leq & C\left|\int_{0}^{1} \bar{\rho}^{k+1} u_{x}^{k} \bar{\theta}^{k+1} d x\right|+C\left|\int_{0}^{1} \bar{\theta}^{k+1} u_{x}^{k} \bar{\theta}^{k+1} d x\right| \\
\leq & \left.\eta||_{x}^{k+1}\right|_{L^{2}} ^{2}+C_{\eta}\left|u_{x}^{k}\right|_{L^{2}}^{2}\left|\bar{\rho}^{k+1}\right|_{L^{2}}^{2} \\
& +C\left|u_{x}^{k}\right|_{L^{2}}\left|\sqrt{\rho^{k+1}} \bar{\theta}^{k+1}\right|_{L^{2}}^{2}, \\
I_{42}= & -\int_{0}^{1} \psi^{k} \bar{u}_{x}^{k} \bar{\theta}^{k+1} d x \leq \eta\left|\bar{\theta}_{x}^{k+1}\right|_{L^{2}}^{2}+C_{\eta}\left|\bar{u}_{x}^{k}\right|_{L^{2}}^{2} .
\end{aligned}
$$

Similarly,

$$
\begin{aligned}
I_{5} & =\int_{0}^{1}\left[\left(u_{x}^{k}\right)^{2}-\left(u_{x}^{k-1}\right)^{2}\right] \bar{\theta}^{k+1} d x \\
& \leq\left|\bar{u}_{x}^{k}\right|_{L^{2}}\left|\theta_{x}^{k+1}\right|_{L^{2}}\left(\left|u_{x}^{k}\right|_{L^{2}}+\left|u_{x}^{k-1}\right|_{L^{2}}\right) \\
& \leq \eta\left|\bar{\theta}_{x}^{k+1}\right|_{L^{2}}^{2}+C_{\eta}\left(\left|u_{x}^{k}\right|_{L^{2}}^{2}+\left|u_{x}^{k-1}\right|_{L^{2}}^{2}\right)\left|\bar{u}_{x}^{k}\right|_{L^{2}}^{2} .
\end{aligned}
$$

Using the same method, we have

$$
\begin{aligned}
I_{6}= & -\int_{0}^{1}\left[\left(\rho^{k+1}\right)^{2} \varphi_{2}^{k+1} u_{x}^{k}-\left(\rho^{k}\right)^{2} \varphi_{2}^{k} u_{x}^{k-1}\right] \bar{\theta}^{k+1} d x \\
= & -\int_{0}^{1} \bar{\rho}^{k+1}\left(\rho^{k+1}+\rho^{k}\right) \varphi_{2}^{k+1} u_{x}^{k} \bar{\theta}^{k+1} d x \\
& -\int_{0}^{1}\left(\rho^{k}\right)^{2} \bar{\varphi}_{2}^{k+1} u_{x}^{k} \bar{\theta}^{k+1} d x \\
& -\int_{0}^{1}\left(\rho^{k}\right)^{2} \varphi_{2}^{k} u_{x}^{k} \bar{\theta}^{k+1} d x=\sum_{j=1}^{3} I_{6 j} .
\end{aligned}
$$

Here,

$$
\begin{aligned}
I_{61}= & \int_{0}^{1} \bar{\rho}^{k+1}\left(\rho^{k+1}+\rho^{k}\right) \varphi_{2}^{k+1} u_{x}^{k} \bar{\theta}^{k+1} d x \\
\leq & \eta\left|\bar{\theta}_{x}^{k+1}\right|_{L^{2}}^{2} \\
& +\left.C_{\eta}\left(\left|\rho_{x}^{k+1}\right|_{L^{2}}+\left.\left|\rho_{x}^{k}\right|\right|_{L^{2}}\right)\left|u_{x}^{k}\right|\right|_{L^{2}}\left|\bar{\rho}^{k+1}\right|_{L^{2}}^{2}, \\
I_{62}= & \int_{0}^{1}\left(\rho^{k}\right)^{2} \bar{\varphi}_{2}^{k+1} u_{x}^{k} \bar{\theta}^{k+1} d x \\
= & C\left|\int_{0}^{1}\left(\rho^{k}\right)^{2} \bar{\rho}^{k+1} u_{x}^{k} \bar{\theta}^{k+1} d x\right| \\
& +C\left|\int_{0}^{1}\left(\rho^{k}\right)^{2} \bar{\theta}^{k+1} u_{x}^{k} \bar{\theta}{ }^{k+1} d x\right| \\
\leq & \eta\left|\bar{\theta}_{x}^{k+1}\right|_{L^{2}}^{2}+C_{\eta}\left|u_{x}^{k}\right|_{L^{2}}^{2}\left|\rho_{x}^{k}\right|_{L^{2}}^{4}\left|\rho^{k+1}\right|_{L^{2}}^{2} \\
& +C\left|u_{x}^{k}\right|_{L^{2}}\left|\rho_{x}^{k}\right|_{L^{2}}^{2}\left|\sqrt{\rho^{k+1}} \bar{\theta}^{k+1}\right|_{L^{2}}^{2}, \\
I_{63}= & \int_{0}^{1}\left(\rho^{k}\right)^{2} \varphi_{2}^{k} \bar{u}_{x}^{k} \bar{\theta}^{k+1} d x \\
\leq & \left.\eta||_{x}^{k+1}\right|_{L^{2}} ^{2}+C_{\eta}\left|\rho_{x}^{k}\right|_{L^{2}}^{4}\left|\bar{u}_{x}^{k}\right|_{L^{2}}^{2} .
\end{aligned}
$$


Substituting $I_{j}(j=1,2, \ldots, 6)$ into (105), after choosing $\eta=\mu_{1}^{(p-2) / 2} / 26$, we get

$$
\begin{aligned}
& \frac{1}{2} \frac{d}{d t}\left|\sqrt{\rho^{k+1} \varphi_{1}^{k+1}} \bar{\theta}^{k+1}\right|_{L^{2}}^{2}+\frac{1}{2} \mu_{1}^{(p-2) / 2}\left|\bar{\theta}_{x}^{k+1}\right|_{L^{2}}^{2} \leq\left|\bar{u}_{x}^{k}\right|_{L^{2}}^{2} \\
& \cdot C\left(1+\left|u_{x}^{k}\right|_{L^{2}}^{2}+\left|u_{x}^{k-1}\right|_{L^{2}}^{2}+\left|\rho_{x}^{k}\right|_{L^{2}}^{4}\right)+\left|\bar{\rho}^{k+1}\right|_{L^{2}}^{2} \\
& +C\left[\left|\theta_{x t}^{k}\right|_{L^{2}}^{2}+\left|\rho_{x}^{k}\right|_{L^{2}}^{2}\left|\theta_{x t}^{k}\right|_{L^{2}}^{2}+\left|u_{x}^{k}\right|_{L^{2}}^{2}\left|\theta_{x t}^{k+1}\right|_{L^{2}}^{2}\right. \\
& +\left|u_{x}^{k}\right|_{L^{2}}^{2}\left|\rho_{x}^{k}\right|_{L^{2}}^{2}\left|\theta_{x t}^{k+1}\right|_{L^{2}}^{2}+\left|u_{x}^{k}\right|_{L^{2}}^{2} \\
& \left.+\left(\left|\rho_{x}^{k+1}\right|_{L^{2}}+\left|\rho_{x}^{k}\right|_{L^{2}}\right)\left|u_{x}^{k}\right|_{L^{2}}+\left|u_{x}^{k}\right|_{L^{2}}^{2}\left|\rho_{x}^{k}\right|_{L^{2}}^{4}\right] \\
& +\left|\sqrt{\rho^{k+1}} \bar{\theta}^{k+1}\right|_{L^{2}}^{2} \cdot C\left(\left.\left|\rho_{x}^{k+1}\right|\right|_{L^{2}}\left|u_{x}^{k}\right|_{L^{2}}^{2}+\left|\theta_{x t}^{k+1}\right|_{L^{2}}^{2}\right. \\
& +\left|\rho_{x}^{k+1}\right|_{L^{2}}^{3}\left|u_{x}^{k}\right|_{L^{2}}^{2}+\left|\rho_{x}^{k}\right|_{L^{2}}\left|\theta_{x t}^{k}\right|_{L^{2}}+\left|\rho_{x}^{k}\right|_{L^{2}}^{2}\left|\theta_{x t}^{k+1}\right|_{L^{2}}^{2} \\
& +\left|\rho_{x}^{k}\right|_{L^{2}}^{2}\left|\theta_{x t}^{k+1}\right|_{L^{2}}^{2}+\left|\rho_{x}^{k}\right|_{L^{2}}^{2}\left|u_{x}^{k-1}\right|_{L^{2}}^{2}+\left|u_{x}^{k}\right|_{L^{2}} \\
& \left.+\left|u_{x}^{k}\right|_{L^{2}}\left|\rho_{x}^{k}\right|_{L^{2}}^{2}\right) \cdot
\end{aligned}
$$

Thus, we get

$$
\begin{aligned}
& \frac{\sqrt{\delta}}{2} \frac{d}{d t}\left|\sqrt{\rho^{k+1}} \bar{\theta}^{k+1}\right|_{L^{2}}^{2}+\frac{1}{2} \mu_{1}^{(p-2) / 2}\left|\bar{\theta}_{x}^{k+1}\right|_{L^{2}}^{2} \\
& \leq \widetilde{C}\left|\bar{u}_{x}^{k}\right|_{L^{2}}^{2}+D^{k}(t)\left(\left|\bar{\rho}^{k+1}\right|_{L^{2}}^{2}+\left|\sqrt{\rho^{k+1}} \bar{\theta}^{k+1}\right|_{L^{2}}^{2}\right) .
\end{aligned}
$$

There $D^{k}(t) \subset L^{1}\left(0, T_{1}\right), \int_{0}^{1} D^{k}(t) \leq \widetilde{C}+\widetilde{C} t$. obtain

Multiplying (74) by $\bar{u}^{k+1}$ and integrating it over $(0,1)$, we

$$
\begin{aligned}
\frac{1}{2} \frac{d}{d t} \int_{0}^{1} \rho^{k+1}\left|\bar{u}^{k+1}\right|^{2} d x \\
\quad+\int_{0}^{1}\left(H_{q}^{\mu_{2}} u^{k+1}-H_{q}^{\mu_{2}} u^{k}\right) \bar{u}^{k+1} d x \\
=-\int_{0}^{1}\left(\psi_{x}^{k+1}-\psi_{x}^{k}\right) \bar{u}^{k+1} d x \\
\quad+\int_{0}^{1} \bar{\rho}^{k+1}\left(-u_{t}^{k}-u^{k-1} u_{x}^{k}\right) \bar{u}^{k+1} d x \\
\quad-\int_{0}^{1} \rho^{k+1} \bar{u}^{k} u_{x}^{k} \bar{u}^{k+1} d x .
\end{aligned}
$$

We use the Sobolev inequality, Young's inequality, and Lemma 3, and we obtain

$$
\begin{aligned}
& I_{1}=-\int_{0}^{1}\left(\psi_{x}^{k+1}-\psi_{x}^{k}\right) \bar{u}^{k+1} d x d x=-\int_{0}^{1} \bar{\psi}^{k+1} \bar{u}^{k+1} d x \\
& \leq C\left|\int_{0}^{1} \bar{\rho}^{k+1} \bar{u}^{k+1} d x\right|+C\left|\int_{0}^{1} \bar{\theta}^{k+1} \bar{u}^{k+1} d x\right| \\
& \leq \eta\left|\bar{u}_{x}^{k+1}\right|_{L^{2}}^{2}+C_{\eta}\left|\bar{\rho}^{k+1}\right|_{L^{2}}^{2}+\eta\left|\bar{u}_{x}^{k+1}\right|_{L^{2}}^{2} \\
& +C_{\eta}\left|\sqrt{\rho^{k+1}} \bar{\theta}^{k+1}\right|_{L^{2}}^{2}, \\
& I_{2}=-\int_{0}^{1} \bar{\rho}^{k+1} u_{t}^{k} \bar{u}^{k+1} d x \leq\left|\bar{\rho}^{k+1}\right|_{L^{2}}\left|u_{t}^{k}\right|_{L^{\infty}}\left|\bar{u}_{x}^{k+1}\right|_{L^{2}} \\
& \leq \eta\left|\bar{u}_{x}^{k+1}\right|_{L^{2}}^{2}+C_{\eta}\left|u_{x t}^{k}\right|_{L^{2}}^{2}\left|\bar{\rho}^{k+1}\right|_{L^{2}}^{2}, \\
& I_{3}=-\int_{0}^{1} \bar{\rho}^{k+1} u^{k-1} u_{x}^{k} \bar{u}^{k+1} d x \\
& \leq\left|\bar{\rho}^{k+1}\right|_{L^{2}}\left|u^{k-1}\right|_{L^{\infty}}\left|u_{x}^{k}\right|_{L^{2}}\left|\bar{u}^{k+1}\right|_{L^{\infty}} \\
& \leq\left|\bar{\rho}^{k+1}\right|_{L^{2}}\left|u_{x}^{k-1}\right|_{L^{2}}\left|u_{x}^{k}\right|_{L^{2}}\left|\bar{u}_{x}^{k+1}\right|_{L^{2}} \\
& \leq \eta\left|\bar{u}_{x}^{k+1}\right|_{L^{2}}^{2}+C_{\eta}\left|u_{x}^{k-1}\right|_{L^{2}}^{2}\left|u_{x}^{k}\right|_{L^{2}}^{2}\left|\bar{\rho}^{k+1}\right|_{L^{2}}^{2}, \\
& I_{4}=-\int_{0}^{1} \rho^{k+1} \bar{u}^{k} u_{x}^{k} \bar{u}^{k+1} d x \\
& \leq C\left|\rho^{k+1}\right|_{L^{\infty}}^{1 / 2}\left|\sqrt{\rho^{k+1}} \bar{u}^{k+1}\right|_{L^{2}}\left|u_{x}^{k}\right|_{L^{2}}\left|\bar{u}_{x}^{k}\right|_{L^{2}} \\
& \leq \varepsilon\left|\bar{u}_{x}^{k}\right|_{L^{2}}^{2}+C_{\varepsilon}\left|\rho_{x}^{k+1}\right|_{L_{2}}\left|u_{x}^{k}\right|_{L^{2}}^{2}\left|\sqrt{\rho^{k+1}} \bar{u}^{k+1}\right|_{L^{2}}^{2} .
\end{aligned}
$$

Substituting $I_{j}(j=1,2, \ldots, 4)$ into (91), after choosing $\eta=\mu_{2}^{(q-2) / 2} / 8$,

$$
\begin{gathered}
\frac{1}{2} \frac{d}{d t} \int_{0}^{1} \rho^{k+1}\left|\bar{u}^{k+1}\right|^{2} d x+\frac{1}{2} \mu_{2}^{(q-2) / 2}\left|\bar{u}_{x}^{k+1}\right|_{L^{2}}^{2} \\
\leq\left|\bar{\rho}^{k+1}\right|_{L^{2}}^{2} \cdot C\left(1+\left|u_{x t}^{k}\right|_{L^{2}}^{2}+\left|u_{x}^{k-1}\right|_{L^{2}}^{2}\left|u_{x}^{k}\right|_{L^{2}}^{2}\right) \\
+\left|\sqrt{\rho^{k+1}} \bar{\theta}^{k+1}\right|_{L^{2}}^{2} \cdot C_{\varepsilon}\left|\bar{u}_{x}^{k}\right|_{L^{2}}^{2} \\
\quad+C_{\varepsilon}\left|\rho_{x}^{k+1}\right|_{L_{2}}\left|u_{x}^{k}\right|_{L^{2}}^{2}\left|\sqrt{\rho^{k+1}} \bar{u}^{k+1}\right|_{L^{2}}^{2} .
\end{gathered}
$$

Thus, we get

$$
\begin{aligned}
& \frac{d}{d t}\left|\bar{\rho}^{k+1}\right|_{L^{2}}^{2} \\
& \leq C\left|\bar{\rho}^{k+1}\right|_{L^{2}}^{2}\left|u_{x}^{k}\right|_{L^{\infty}}+\left|\rho^{k}\right|_{H^{1}}\left|\bar{u}_{x}^{k}\right|_{L^{2}}\left|\bar{\rho}^{k+1}\right|_{L^{2}} \\
& \leq B_{\eta}^{k}(t)\left|\bar{\rho}^{k+1}\right|_{L^{2}}^{2}+\eta\left|\bar{u}_{x}^{k}\right|_{L^{2}}^{2},
\end{aligned}
$$




$$
\begin{aligned}
& \int_{0}^{1} B_{\eta}^{k}(t) \leq \widetilde{C} t, \\
& \frac{1}{2} \frac{d}{d t}\left|\sqrt{\rho^{k+1}} \bar{\theta}^{k+1}\right|_{L^{2}}^{2}+\frac{1}{2} \mu_{1}^{(p-2) / 2} \int_{0}^{1}\left|\bar{\theta}_{x}^{k+1}\right|_{L^{2}}^{2} d x \\
& \leq \widetilde{C}\left|\bar{u}_{x}^{k}\right|_{L^{2}}^{2}+D^{k}(t)\left(\left|\bar{\rho}^{k+1}\right|_{L^{2}}^{2}+\left|\sqrt{\rho^{k+1}} \bar{\theta}^{k+1}\right|_{L^{2}}^{2}\right), \\
& \int_{0}^{1} D^{k}(t) \leq \widetilde{C}+\widetilde{C} t, \\
& \frac{1}{2} \frac{d}{d t} \int_{0}^{1} \rho^{k+1}\left|\bar{u}^{k+1}\right|^{2} d x+\frac{1}{2} \mu_{2}^{(q-2) / 2}\left|\bar{u}_{x}^{k+1}\right|_{L^{2}}^{2} \\
& \leq E_{\varepsilon}^{k}(t)\left(\left|\bar{\rho}^{k+1}\right|_{L^{2}}^{2}+\left|\sqrt{\rho^{k+1}} \bar{\theta}^{k+1}\right|_{L^{2}}^{2}+\left|\sqrt{\rho^{k+1}} \bar{u}^{k+1}\right|_{L^{2}}^{2}\right) \\
& \quad+\varepsilon\left|\bar{u}_{x}^{k}\right|_{L^{2}}^{2} \cdot
\end{aligned}
$$

There $E_{\varepsilon}^{k}(t) \subset L^{1}\left(0, T_{1}\right), \int_{0}^{1} E_{\varepsilon}^{k}(t) \leq \widetilde{C}+\widetilde{C}_{\varepsilon} t$. Using (94), denote

$$
\begin{gathered}
\Psi^{k+1}=\left|\bar{\rho}^{k+1}\right|_{L^{2}}^{2}+\frac{\varepsilon}{2}\left|\sqrt{\rho^{k+1}} \bar{\theta}^{k+1}\right|_{L^{2}}^{2} \\
+\frac{1}{2}\left|\sqrt{\rho^{k+1}} \bar{u}^{k+1}\right|_{L^{2}}^{2} .
\end{gathered}
$$

There $F_{\varepsilon}^{k}(t) \subset L^{1}\left(0, T_{1}\right), \int_{0}^{1} F_{\varepsilon}^{k}(t) \leq \widetilde{C}+\widetilde{C}_{\varepsilon} t$.

Thus, we get

$$
\begin{gathered}
\frac{d}{d t} \Psi^{k+1}(t)+\frac{\varepsilon}{2 \sqrt{\delta}} \mu_{1}^{(p-2) / 2}\left|\bar{\theta}_{x}^{k+1}\right|_{L^{2}}^{2} \\
+\frac{1}{2} \mu_{2}^{(q-2) / 2}\left|\bar{u}_{x}^{k+1}\right|_{L^{2}}^{2} \\
\leq F_{\varepsilon}^{k}(t) \cdot \Psi^{k+1}(t)+\widetilde{C} \cdot \varepsilon \cdot\left|\bar{u}_{x}^{k}\right|_{L^{2}}^{2} .
\end{gathered}
$$

By Gronwall's inequality, noting that $\Psi^{k+1}(t)=0$, we get

$$
\begin{aligned}
& \Psi^{k+1}(t)+\frac{\varepsilon}{2 \sqrt{\delta}} \mu_{1}^{(p-2) / 2} \int_{0}^{t}\left|\bar{\theta}_{x}^{k+1}\right|_{L^{2}}^{2}(s) d s \\
& +\frac{1}{2} \mu_{2}^{(q-2) / 2} \int_{0}^{t}\left|\bar{u}_{x}^{k+1}\right|_{L^{2}}^{2}(s) d s \leq \exp \left\{\int_{0}^{t} F_{\varepsilon}^{k}(t) d t\right\} \\
& \cdot \int_{0}^{t} \widetilde{C} \cdot \varepsilon \cdot\left|\bar{u}_{x}^{k}\right|_{L^{2}}^{2}(s) d s \leq \exp \left\{\widetilde{C}+\widetilde{C}_{\varepsilon} t\right\} \\
& \cdot \int_{0}^{t} \widetilde{C} \cdot \varepsilon \cdot\left|\bar{u}_{x}^{k}\right|_{L^{2}}^{2}(s) d s .
\end{aligned}
$$

We can obtain

$$
\begin{aligned}
\widetilde{C} \cdot \varepsilon \cdot \exp \{\widetilde{C}\} & =\frac{1}{4}, \\
\exp \left\{\widetilde{C}_{\varepsilon} \cdot T_{7}\right\} & =\mu_{2}^{(q-2) / 2},
\end{aligned}
$$

and thus we can get

$$
\begin{aligned}
& \sum_{k=1}^{K} \Psi^{k+1}(t)+\sum_{k=1}^{K} \int_{0}^{T^{*}}\left(\left|\bar{\theta}_{x}^{k+1}(s)\right|_{L^{2}}^{2}+\left|\bar{u}_{x}^{k+1}(s)\right|_{L^{2}}^{2}\right) d s \\
& \leq \widetilde{C}<+\infty .
\end{aligned}
$$

There, $T^{*}=\min \left\{T_{6}, T_{7}\right\}$.

Therefore, we conclude that the full sequence $\left(\rho^{k}, u^{k}, \theta^{k}\right)$ converges to a limit $(\rho, u, \theta)$ in the following strong sense:

$$
\begin{aligned}
& \rho^{k} \longrightarrow \rho \quad \text { in } L^{\infty}\left(0, T^{*} ; L^{2}\right), \\
& u^{k} \longrightarrow u \quad \text { in } L^{\infty}\left(0, T^{*} ; L^{2}\right) \cap L^{2}\left(0, T^{*} ; H_{0}^{1}\right), \\
& \theta^{k} \longrightarrow \theta \quad \text { in } L^{\infty}\left(0, T^{*} ; L^{2}\right) \cap L^{2}\left(0, T^{*} ; H_{0}^{1}\right),
\end{aligned}
$$

for $T^{*}=\min \left[T_{1}, T_{2}, T_{3}, T_{4}, T_{5}, T_{6}, T_{7}\right]$, and we get

$$
\begin{aligned}
& \sup _{0 \leq s \leq T^{*}}\left|u_{x}\right|_{L^{2}}(s)+\int_{0}^{T^{*}}\left|u_{x t}^{k}\right|_{L^{2}}^{2}(s) d s \leq c_{1}, \\
& \sup _{0 \leq s \leq T^{*}}\left|u_{x x}\right|_{L^{2}}(s) \leq c_{2}, \\
& \sup _{0 \leq s \leq T^{*}}\left|\theta_{x}\right|_{L^{2}}^{2}(s)+\left|\sqrt{\rho^{k}} \theta_{t}^{k}(t)\right|_{L^{2}}^{2}(s) \\
& +\int_{0}^{T^{*}}\left|\theta_{x t}^{k}\right|_{L^{2}}^{2}(s) d s \leq C c_{1}^{5}, \\
& \sup _{0 \leq s \leq T^{*}}\left|u_{x}\right|_{L^{2}}^{2}(s)+\left|\sqrt{\rho^{k}} u_{t}^{k}(t)\right|_{L^{2}}^{2}(s) \\
& +\int_{0}^{T^{*}}\left|u_{x t}\right|_{L^{2}}^{2}(s) d s \leq c_{1}, \\
& \sup _{0 \leq s \leq T^{*}}\left|\theta_{x x}\right|_{L^{2}}(s)<C c_{1}^{4} c_{2}, \\
& \sup _{0 \leq s \leq T^{*}}|\rho(t)|_{H^{1}}(s) \leq C c_{0}, \\
& \sup _{0 \leq s \leq T^{*}}\left|\rho_{t}(t)\right|_{L^{2}}(s) \leq C c_{1}^{2} .
\end{aligned}
$$

\section{Proof of the Main Theorem}

4.1. Existence $(k \rightarrow \infty)$. In this part, we need to prove that $(\rho, u, \theta)$ is a solution of problem

$$
\begin{aligned}
& \rho_{t}+(\rho u)_{x}=0, \quad(x, t) \in \Omega_{T}, \\
& (\rho e)_{t}+(\rho u e)_{x}-\left[\left(\theta_{x}^{2}+\mu\right)^{(p-2) / 2} \theta_{x}\right]_{x}+\pi u_{x} \\
& =\left|u_{x}\right|^{2}, \quad(x, t) \in \Omega_{T},
\end{aligned}
$$


14

Advances in Mathematical Physics

$$
\begin{aligned}
& (\rho u)_{t}+\left(\rho u^{2}\right)_{x}-\left[\left(u_{x}^{2}+\mu_{2}\right)^{(q-2) / 2} u_{x}\right]_{x}+\pi_{x}=0, \\
& \quad(x, t) \in \Omega_{T}, \\
& \pi=\varphi(\theta, \rho), \quad(x, t) \in \Omega_{T}, \\
& e=\psi(\theta, \rho), \quad(x, t) \in \Omega_{T},
\end{aligned}
$$

with the initial and boundary conditions

$$
\begin{aligned}
\left.\rho\right|_{t=0} & =\rho_{0} \geq 0, \\
\left.u\right|_{t=0} & =u_{0}, \\
\left.\theta\right|_{t=0} & =\theta_{0}, \\
u(0, t) & =u(1, t)=0, \\
\theta(0, t) & =\theta(1, t)=0
\end{aligned}
$$

and also satisfy the integral identity after the $k \rightarrow \infty$ process. Finally, we need to show that the solution satisfies the regular properties in Definition 1. Since the abovementioned can be obtained by the same method as in [15], we omit it for brevity.

4.2. Uniqueness. Let $\left(\rho^{1}, u^{1}, \theta^{1}\right),\left(\rho^{2}, u^{2}, \theta^{2}\right)$ be two solutions of problem (3)-(4). After substituting into (7), respectively, we define

$$
\begin{aligned}
& \bar{\rho}=\rho^{1}-\rho^{2}, \\
& \bar{u}=u^{2}-u^{1}, \\
& \bar{\theta}=\theta^{1}-\theta^{2},
\end{aligned}
$$

and then we can obtain

$$
\begin{aligned}
\frac{1}{2} \frac{d}{d t} \int_{0}^{1} \rho^{1} \varphi_{1}^{1}|\bar{\theta}|^{2} d x+\int_{0}^{1}\left(H_{p}^{\mu_{1}} \theta^{1}-H_{p}^{\mu_{1}} \theta^{2}\right) \bar{\theta} d x \\
=\frac{1}{2} \int_{0}^{1}\left(\rho^{1} \varphi_{1}^{1}\right)_{t}|\bar{\theta}|^{2} d x-\int_{0}^{1} \rho^{1} \varphi_{1}^{1} \theta_{t}^{2} \bar{\theta} d x \\
\quad+\int_{0}^{1} \rho^{2} \varphi_{1}^{2} \theta_{t}^{2} \bar{\theta} d x \\
\quad-\int_{0}^{1}\left[\rho^{1} \varphi_{1}^{1} u^{1} \theta_{x}^{1}-\rho^{2} \varphi_{1}^{2} u^{2} \theta_{x}^{2}\right] \bar{\theta} d x \\
\quad-\int_{0}^{1}\left[\psi^{1} u_{x}^{1}-\psi^{2} u_{x}^{2}\right] \bar{\theta} d x \\
\quad+\int_{0}^{1}\left[\left(u_{x}^{1}\right)^{2}-\left(u_{x}^{2}\right)^{2}\right] \bar{\theta} d x \\
\quad+\int_{0}^{1}\left[\left(\rho^{1}\right)^{2} \varphi_{2}^{1} u_{x}^{1}-\left(\rho^{2}\right)^{2} \varphi_{2}^{2} u_{x}^{2}\right] \bar{\theta} d x=\sum_{j=1}^{6} I_{j},
\end{aligned}
$$

since

$$
\begin{gathered}
\int_{0}^{t} \int_{0}^{1}\left(\left|\theta_{x}^{1}\right|^{p-2} \theta_{x}^{1}-\left|\theta_{x}^{2}\right|^{p-2} \theta_{x}^{2}\right) \bar{\theta}_{x} d x \\
\geq \mu_{1}^{(p-2) / 2} \int_{0}^{t}\left|\bar{\theta}_{x}\right|_{L^{2}}^{2} d s .
\end{gathered}
$$

Using the Sobolev inequality, Young's inequality, and Lemma 3, we obtain

$$
\begin{aligned}
I_{1}= & \frac{1}{2} \int_{0}^{1}\left[\rho^{1} \varphi_{1}^{1}\right]_{t}|\bar{\theta}|^{2} d x \\
= & \frac{1}{2} \int_{0}^{1} \rho_{t}^{1} \varphi_{1}^{1}|\bar{\theta}|^{2} d x+\frac{1}{2} \int_{0}^{1} \rho^{1} \varphi_{11}^{1} \theta_{t}^{1}|\bar{\theta}|^{2} d x \\
& +\frac{1}{2} \int_{0}^{1} \rho^{1} \varphi_{12}^{1} \rho_{t}^{1}|\bar{\theta}|^{2} d x=\sum_{j=1}^{3} I_{1 j} .
\end{aligned}
$$

Here,

$$
\begin{aligned}
I_{11} & =\frac{1}{2} \int_{0}^{1} \rho_{t}^{1} \varphi_{1}^{1}\left|\bar{\theta}^{1}\right|^{2} d x \leq\left. C\left|\int_{0}^{1} \rho_{t}^{1}\right| \bar{\theta}\right|^{2} d x \mid \\
& =C\left|\int_{0}^{1} \rho^{1} u^{1} \bar{\theta} \bar{\theta}_{x} d x\right| \\
& \leq C\left|\rho^{1}\right|_{L^{\infty}}^{1 / 2}\left|\sqrt{\rho^{1}} \bar{\theta}^{1}\right|_{L^{2}}\left|u^{1}\right|_{L^{\infty}}\left|\bar{\theta}_{x}\right|_{L^{2}} \\
& \leq \eta\left|\bar{\theta}_{x}\right|_{L^{2}}^{2}+C_{\eta}\left|\rho_{x}^{1}\right|_{L^{2}}\left|u_{x}^{1}\right|_{L^{2}}^{2}\left|\sqrt{\rho^{1}} \bar{\theta}\right|_{L^{2}}^{2}, \\
I_{12} & =\frac{1}{2} \int_{0}^{1} \rho^{1} \varphi_{11}^{1} \theta_{t}^{1}|\bar{\theta}|^{2} d x \leq\left. C\left|\int_{0}^{1} \rho^{1} \theta_{t}^{1}\right| \bar{\theta}^{1}\right|^{2} d x \mid \\
& \leq C\left|\theta_{t}^{1}\right|_{L^{\infty}}\left|\sqrt{\rho^{1}} \bar{\theta}\right|_{L^{2}}^{2} \leq C\left|\theta_{x t}^{1}\right|_{L^{2}}\left|\sqrt{\rho^{1}} \bar{\theta}\right|_{L^{2}}^{2}, \\
I_{13} & =\frac{1}{2} \int_{0}^{1} \rho^{1} \varphi_{12}^{1} \rho_{t}^{1}|\bar{\theta}|^{2} d x \\
& \leq\left. C\left|\rho^{1}\right|_{L^{\infty}}\left|\int_{0}^{1} \rho_{t}^{1}\right| \bar{\theta}^{1}\right|^{2} d x \mid \\
& \leq \eta\left|\bar{\theta}_{x}\right|_{L^{2}}^{2}+C_{\eta}\left|\rho_{x}^{1}\right|_{L^{2}}^{3}\left|u_{x}^{1}\right|_{L^{2}}^{2}\left|\sqrt{\rho^{1}} \bar{\theta}\right|_{L^{2}}^{2} .
\end{aligned}
$$

In the same way,

$$
\begin{aligned}
I_{2}= & -\int_{0}^{1} \rho^{1} \varphi_{1}^{1} \theta_{t}^{2} \bar{\theta} d x+\int_{0}^{1} \rho^{2} \varphi_{1}^{2} \theta_{t}^{2} \bar{\theta} d x \\
= & -\int_{0}^{1} \bar{\rho} \varphi_{1}^{1} \theta_{t}^{2} \bar{\theta} d x+\int_{0}^{1} \rho^{2} \bar{\varphi}_{1}^{1} \theta_{t}^{2} \bar{\theta} d x \\
\leq & C\left|\int_{0}^{1} \bar{\rho}^{1} \theta_{t}^{2} \bar{\theta} d x\right|+C\left|\int_{0}^{1} \rho^{2} \bar{\rho} \theta_{t}^{2} \bar{\theta} d x\right| \\
& +C\left|\int_{0}^{1} \rho^{2} \bar{\theta} \theta_{t}^{2} \bar{\theta} d x\right|=\sum_{j=1}^{3} I_{2 j} .
\end{aligned}
$$


Advances in Mathematical Physics

15

Here,

$$
\begin{aligned}
I_{21} & =C\left|\int_{0}^{1} \bar{\rho}^{1} \theta_{t}^{2} \bar{\theta}^{1} d x\right| \leq C|\bar{\rho}|_{L^{2}}\left|\theta_{x t}^{2}\right|_{L^{2}}\left|\bar{\theta}_{x}\right|_{L^{2}} \\
& \leq \eta\left|\bar{\theta}_{x}\right|_{L^{2}}^{2}+C_{\eta}\left|\theta_{x t}^{2}\right|_{L^{2}}^{2}|\bar{\rho}|_{L^{2}}^{2}, \\
I_{22} & =C\left|\int_{0}^{1} \rho^{2} \bar{\rho}^{1} \theta_{t}^{2} \bar{\theta}^{1} d x\right| \\
& \leq C\left|\rho_{x}^{2}\right|_{L^{2}}|\bar{\rho}|_{L^{2}}\left|\theta_{x t}^{2}\right|_{L^{2}}\left|\bar{\theta}_{x}\right|_{L^{2}} \\
& \leq \eta\left|\bar{\theta}_{x}\right|_{L^{2}}^{2}+C_{\eta}\left|\rho_{x}^{2}\right|_{L^{2}}^{2}\left|\theta_{x t}^{2}\right|_{L^{2}}^{2}|\bar{\rho}|_{L^{2}}^{2}, \\
I_{23} & =C\left|\int_{0}^{1} \rho^{k} \bar{\theta} \theta_{t}^{2} \bar{\theta} d x\right| \leq C\left|\rho_{x}^{2}\right|_{L^{2}}\left|\theta_{x t}^{2}\right|_{L^{2}}\left|\sqrt{\rho^{1}} \bar{\theta}\right|_{L^{2}}^{2} .
\end{aligned}
$$

By the same token,

$$
\begin{aligned}
I_{3}= & -\int_{0}^{1}\left[\rho^{1} \varphi_{1}^{1} u^{1} \theta_{x}^{1}-\rho^{2} \varphi_{1}^{2} u^{2} \theta_{x}^{2}\right] \bar{\theta} d x \\
= & -\int_{0}^{1} \bar{\rho} \varphi_{1}^{1} u^{1} \theta_{x}^{1} \bar{\theta} d x-\int_{0}^{1} \rho^{2} \bar{\varphi}_{1} u^{1} \theta_{x}^{1} \bar{\theta} d x \\
& -\int_{0}^{1} \rho^{2} \varphi_{1}^{2} \bar{u} \theta_{x}^{1} \bar{\theta} d x-\int_{0}^{1} \rho^{2} \varphi_{1}^{2} u^{2} \bar{\theta}_{x} \bar{\theta} d x=\sum_{j=1}^{4} I_{3 j} .
\end{aligned}
$$

Here,

$$
\begin{aligned}
I_{31}= & -\int_{0}^{1} \bar{\rho} \varphi_{1}^{1} u^{1} \theta_{x}^{1} \bar{\theta} d x \leq C|\bar{\rho}|_{L^{2}}\left|u_{x}^{1}\right|_{L^{2}}\left|\theta_{x t}^{1}\right|_{L^{2}}\left|\bar{\theta}_{x}\right|_{L^{2}} \\
\leq & \eta\left|\bar{\theta}_{x}\right|_{L^{2}}^{2}+C_{\eta}\left|u_{x}^{1}\right|_{L^{2}}^{2}\left|\theta_{x t}^{1}\right|_{L^{2}}^{2}|\bar{\rho}|_{L^{2}}^{2}, \\
I_{32}= & -\int_{0}^{1} \rho^{2} \bar{\varphi}_{1} u^{1} \theta_{x}^{1} \bar{\theta} d x \\
\leq & C\left|\int_{0}^{1} \rho^{2} \bar{\rho} u^{1} \theta_{x}^{1} \bar{\theta} d x\right|+C\left|\int_{0}^{1} \rho^{2} \bar{\theta} u^{1} \theta_{x}^{1} \bar{\theta} d x\right| \\
\leq & \eta\left|\bar{\theta}_{x}\right|_{L^{2}}^{2}+C_{\eta}\left|u_{x}^{1}\right|_{L^{2}}^{2}\left|\rho_{x}^{2}\right|_{L^{2}}^{2}\left|\theta_{x t}^{1}\right|_{L^{2}}^{2}|\bar{\rho}|_{L^{2}}^{2} \\
& +C\left|u_{x}^{1}\right|_{L^{2}}\left|\rho_{x}^{2}\right|_{L^{2}}\left|\theta_{x t}^{1}\right|_{L^{2}}\left|\sqrt{\rho^{1}} \bar{\theta}\right|_{L^{2}}^{2} \\
I_{33}= & \int_{0}^{1} \rho^{2} \varphi_{1}^{2} \bar{u} \theta_{x}^{1} \bar{\theta} d x \\
\leq & C\left|\bar{u}_{x}\right|_{L^{2}}\left|\rho_{x}^{2}\right|_{L^{2}}\left|\theta_{x t}^{1}\right|_{L^{2}}\left|\sqrt{\rho^{1}} \bar{\theta}\right|_{L^{2}} \\
\leq & C\left|\bar{u}_{x}\right|_{L^{2}}^{2}+C\left|\rho_{x}^{2}\right|_{L^{2}}^{2}\left|\theta_{x t}^{1}\right|_{L^{2}}^{2}\left|\sqrt{\rho^{1}} \bar{\theta}\right|_{L^{2}}^{2},
\end{aligned}
$$

$$
\begin{aligned}
I_{34} & =\int_{0}^{1} \rho^{2} \varphi_{1}^{2} u^{2} \bar{\theta}_{x} \bar{\theta} d x\left(\frac{\rho^{1}}{\rho^{1}}\right) \\
& \leq C\left|\rho^{2}\right|_{L^{\infty}}\left|\sqrt{\rho^{1}} \bar{\theta}\right|_{L^{2}}\left|u^{2}\right|_{L^{\infty}}\left|\bar{\theta}_{x}\right|_{L^{2}} \\
& \leq \eta\left|\bar{\theta}_{x}\right|_{L^{2}}^{2}+C_{\eta}\left|\rho_{x}^{2}\right|_{L^{2}}^{2}\left|u_{x}^{2}\right|_{L^{2}}^{2}\left|\sqrt{\rho^{1}} \bar{\theta}\right|_{L^{2}}^{2} .
\end{aligned}
$$

In the similar way, we have

$$
\begin{aligned}
I_{4} & =-\int_{0}^{1}\left[\psi^{1} u_{x}^{1}-\psi^{2} u_{x}^{2}\right] \bar{\theta} d x \\
& =-\int_{0}^{1} \bar{\psi} u_{x}^{1} \bar{\theta} d x-\int_{0}^{1} \psi^{2} \bar{u} \bar{\theta} d x=\sum_{j=1}^{2} I_{4 j} .
\end{aligned}
$$

Here,

$$
\begin{aligned}
& I_{41}=-\int_{0}^{1} \bar{\psi}^{1} u_{x}^{1} \bar{\theta} d x \\
& \leq C\left|\int_{0}^{1} \bar{\rho}^{1} u_{x}^{1} \bar{\theta}^{1} d x\right|+C\left|\int_{0}^{1} \bar{\theta} u_{x}^{1} \bar{\theta} d x\right| \\
& \leq \eta\left|\bar{\theta}_{x}\right|_{L^{2}}^{2}+C_{\eta}\left|u_{x}^{1}\right|_{L^{2}}^{2}|\bar{\rho}|_{L^{2}}^{2}+C\left|u_{x}^{1}\right|_{L^{2}}\left|\sqrt{\rho^{1}} \bar{\theta}\right|_{L^{2}}^{2}, \\
& I_{42}=-\int_{0}^{1} \psi^{2} \bar{u} \bar{\theta}^{1} d x \leq \eta\left|\bar{\theta}_{x}\right|_{L^{2}}^{2}+C_{\eta}\left|\bar{u}_{x}\right|_{L^{2}}^{2} . \\
& I_{5}=\int_{0}^{1}\left[\left(u_{x}^{1}\right)^{2}-\left(u_{x}^{2}\right)^{2}\right] \bar{\theta} d x \\
& \leq\left|\bar{u}_{x}\right|_{L^{2}}\left|\bar{\theta}_{x}\right|_{L^{2}}\left(\left|u_{x}^{1}\right|_{L^{2}}+\left|u_{x}^{2}\right|_{L^{2}}\right) \\
& \leq \eta\left|\bar{\theta}_{x}\right|_{L^{2}}^{2}+C_{\eta}\left(\left|u_{x}^{1}\right|_{L^{2}}^{2}+\left|u_{x}^{2}\right|_{L^{2}}^{2}\right)\left|\bar{u}_{x}\right|_{L^{2}}^{2} .
\end{aligned}
$$

Using the same method, we obtain

$$
\begin{aligned}
I_{6}= & \int_{0}^{1}\left[\left(\rho^{1}\right)^{2} \varphi_{2}^{1} u_{x}^{1}-\left(\rho^{2}\right)^{2} \varphi_{2}^{2} u_{x}^{2}\right] \bar{\theta} d x \\
= & -\int_{0}^{1} \bar{\rho}\left(\rho^{1}+\rho^{2}\right) \varphi_{2}^{1} u_{x}^{1} \bar{\theta} d x-\int_{0}^{1}\left(\rho^{2}\right)^{2} \bar{\varphi}_{2} u_{x}^{1} \bar{\theta} d x \\
& -\int_{0}^{1}\left(\rho^{2}\right)^{2} \varphi_{2}^{2} \bar{u} \bar{\theta} d x=\sum_{j=1}^{3} I_{6 j} .
\end{aligned}
$$


Here,

$$
\begin{aligned}
I_{61}= & \int_{0}^{1} \bar{\rho}\left(\rho^{2}+\rho^{1}\right) \varphi_{2}^{1} u_{x}^{k} \bar{\theta} d x \\
\leq & \eta\left|\bar{\theta}_{x}\right|_{L^{2}}^{2}+C_{\eta}\left(\left|\rho_{x}^{1}\right|_{L^{2}}+\left.\left|\rho_{x}^{2}\right|\right|_{L^{2}}\right)\left|u_{x}^{1}\right|_{L^{2}}|\bar{\rho}|_{L^{2}}^{2}, \\
I_{62}= & \int_{0}^{1}\left(\rho^{2}\right)^{2} \bar{\varphi}_{2} u_{x}^{1} \bar{\theta} d x \\
= & C\left|\int_{0}^{1}\left(\rho^{2}\right)^{2} \bar{\rho} u_{x}^{1} \bar{\theta} d x\right|+C\left|\int_{0}^{1}\left(\rho^{2}\right)^{2} \bar{\theta} u_{x}^{1} \bar{\theta} d x\right| \\
\leq & \eta\left|\bar{\theta}_{x}\right|_{L^{2}}^{2}+C_{\eta}\left|u_{x}^{1}\right|_{L^{2}}^{2}\left|\rho_{x}^{2}\right|_{L^{2}}^{4}|\bar{\rho}|_{L^{2}}^{2} \\
& +C\left|u_{x}^{1}\right|_{L^{2}}\left|\rho_{x}^{2}\right|_{L^{2}}^{2}\left|\sqrt{\rho^{1}} \bar{\theta}\right|_{L^{2}}^{2}, \\
I_{63}= & \int_{0}^{1}\left(\rho^{2}\right)^{2} \varphi_{2}^{2} \bar{u}_{x} \bar{\theta}^{k+1} d x \\
\leq & \eta\left|\bar{\theta}_{x}\right|_{L^{2}}^{2}+C_{\eta}\left|\rho_{x}^{2}\right|_{L^{2}}^{4}|\bar{u}|_{L^{2}}^{2} .
\end{aligned}
$$

Thus, we have

$$
\begin{aligned}
& \frac{\sqrt{\delta}}{2}\left|\sqrt{\rho^{1}} \bar{\theta}\right|_{L^{2}}^{2}+\frac{\mu_{1}^{(p-2) / 2}}{2} \int_{0}^{t}|\bar{\theta}|_{L^{2}}^{2} d s \\
& \leq \int_{0}^{t} E(t)\left(|\bar{\rho}|_{L^{2}}^{2}+\left|\sqrt{\rho^{1}} \bar{\theta}\right|_{L^{2}}^{2}+\left|\sqrt{\rho^{1}} \bar{u}\right|_{L^{2}}^{2}\right) d s \\
& +\widetilde{C} \int_{0}^{t}\left|\bar{u}_{x}^{k}\right|_{L^{2}}^{2} d s .
\end{aligned}
$$

There $E(t) \in L^{1}\left(0, T^{*}\right), \int_{0}^{1} E(t) \leq \widetilde{C}+\widetilde{C} t$.

In the same way,

$$
\begin{aligned}
& \frac{1}{2}\left|\sqrt{\rho^{1}} \bar{u}\right|_{L^{2}}^{2}+\frac{\mu_{2}^{(p-2) / 2}}{2} \int_{0}^{t}\left|\bar{u}_{x}\right|_{L^{2}}^{2} d s \\
& \leq \int_{0}^{t} F(t)\left(|\bar{\rho}|_{L^{2}}^{2}+\left|\sqrt{\rho^{1}} \bar{\theta}\right|_{L^{2}}^{2}+\left|\sqrt{\rho^{1}} \bar{u}\right|_{L^{2}}^{2}\right) d s .
\end{aligned}
$$

There $F(t) \in L^{1}\left(0, T^{*}\right), \int_{0}^{1} F(t) \leq \widetilde{C}+\widetilde{C} t$. Consider

$$
\left|\bar{\rho}^{1}\right|_{L^{2}}^{2} \leq \int_{0}^{t} B(t)\left(|\bar{\rho}|_{L^{2}}^{2}\right) d s+\eta \int_{0}^{t}\left|\bar{u}_{x}^{k}\right|_{L^{2}}^{2} d s
$$

There $B(t) \in L^{1}\left(0, T^{*}\right), \int_{0}^{1} B(t) \leq \widetilde{C} t$. (120),

With (119) multiplied by $\eta$, combining with (118) and

$$
(\widetilde{C}+1) \eta=\frac{\mu_{2}^{(p-2) / 2}}{4} .
$$

We can get

$$
\begin{aligned}
|\bar{\rho}|_{L^{2}}^{2} & +\left|\sqrt{\rho^{1}} \bar{\theta}\right|_{L^{2}}^{2}+\left|\sqrt{\rho^{1}} \bar{u}\right|_{L^{2}}^{2}+\int_{0}^{t}\left|\bar{\theta}_{x}\right|_{L^{2}}^{2} d s \\
& +\int_{0}^{t}\left|\bar{u}_{x}\right|_{L^{2}}^{2} d s \\
\leq & \int_{0}^{t} G(t)\left(|\bar{\rho}|_{L^{2}}^{2}+\left|\sqrt{\rho^{1}} \bar{\theta}\right|_{L^{2}}^{2}+\left|\sqrt{\rho^{1}} \bar{u}\right|_{L^{2}}^{2}\right) d s .
\end{aligned}
$$

There $G(t) \in L^{1}\left(0, T^{*}\right), \int_{0}^{1} G(t) \leq \widetilde{C} t+\widetilde{C}$.

By Gronwall's inequality, it yields

$$
\begin{aligned}
& u^{1}=u^{2}, \\
& \rho^{1}=\rho^{2}, \\
& \theta^{1}=\theta^{2} .
\end{aligned}
$$

\section{Conflict of Interests}

The authors declare that there is no conflict of interests regarding the publication of this paper.

\section{Acknowledgments}

The research is supported by the National Natural Science Foundation of China Grant no. 11271153. The research is supported by Specialized Research Fund for the Doctoral Program of Higher Education no. 20140101-20161231.

\section{References}

[1] R. B. Bird, C. F. Curtiss, R. C. Armstrong, and O. Hassager, Dynamics of Polymeric Liquids, Volume 2: Kinetic Theory, WileyInterscience, 1987.

[2] G. Astarita and G. Marrucci, Principles of Non-Newtonian Fluid Mechanics, McGraw-Hill, 1974.

[3] M. Moriyama, "On the first initial-boundary value problem for compressible non-newtonian fluid motion," Keio Mathematical Seminar Reports, no. 7, pp. 21-27, 1982.

[4] S. Goel, "Propagation of sound waves in compressible nonNewtonian fluids," Mathematical Seminar, vol. 2, no. 2, pp. 5660, 1961.

[5] V. V. Zhikov and S. E. Pastukhova, "On the solvability of the Navier-Stokes system for a compressible non-Newtonian fluid," Doklady Akademii Nauk, vol. 427, no. 3, pp. 303-307, 2009 (Russian).

[6] A. E. Mamontov, "Global regularity estimates for multidimensional equations of compressible non-Newtonian fluids. NavierStokes equations and related nonlinear problems (Ferrara, 1999)," Annali dell'Università di Ferrara. Sezione VII, vol. 46, pp. 139-160, 2000.

[7] J. Nečas, Les Méthodes Directes en Théorie des Équations Elliptiques, Masson, Paris, France, 1967.

[8] J. L. Lions, Quelques Méthodes de Résolution des Problémes aux Limites non Linéaires, Gauthier-Villiars, Paris, France, 1969. 
[9] O. Ladyzhenskaya, "New equations for the description of the viscous incompressible fluids and solvability in the large of the boundary value problems for them," in Boundary Value Problems of Mathematical Physics V, American Mathematical Society, Providence, RI, USA, 1970.

[10] D. Bothe and J. Prüss, "LP-theory for a class of non-newtonian fluids," SIAM Journal on Mathematical Analysis, vol. 39, no. 2, pp. 379-421, 2007.

[11] Z. Xin, "Blowup of smooth solutions to the compressible Navier-Stokes equation with compact density," Communications on Pure and Applied Mathematics, vol. 51, no. 3, pp. 229-240, 1998.

[12] H. J. Choe and H. Kim, "Strong solutions of the NavierStokes equations for isentropic compressible fluids," Journal of Differential Equations, vol. 190, no. 2, pp. 504-523, 2003.

[13] Y. Cho, H. J. Choe, and H. Kim, "Unique solvability of the initial boundary value problems for compressible viscous fluids," Journal de Mathématiques Pures et Applquées, vol. 83, no. 2, pp. 243-275, 2004.

[14] X. Huang, J. Li, and Z. Xin, "Global well-posedness of classical solutions with large oscillations and vacuum to the threedimensional isentropic compressible Navier-Stokes equations," Communications on Pure and Applied Mathematics, vol. 65, no. 4, pp. 549-585, 2012.

[15] H. Yuan and X. Xu, "Existence and uniqueness of solutions for a class of non-Newtonian fluids with singularity and vacuum," Journal of Differential Equations, vol. 245, no. 10, pp. 2871-2916, 2008.

[16] H. Yuan and Q. Meng, "A strong solution for a class of compressible full non-Newtonian models," Nonlinear Analysis: Theory, Methods \& Applications, vol. 81, pp. 224-235, 2013.

[17] Y. Song, H. Yuan, Y. Chen, and Z. Guo, "Strong solutions for a 1D fluid-particle interaction non-newtonian model: the bubbling regime," Journal of Mathematical Physics, vol. 54, no. 9, Article ID 091501, 12 pages, 2013.

[18] C. Wang and H. Yuan, "Global strong solutions for a class of heat-conducting non-Newtonian fluids with vacuum," Nonlinear Analysis: Real World Applications, vol. 11, no. 5, pp. 36803703, 2010. 


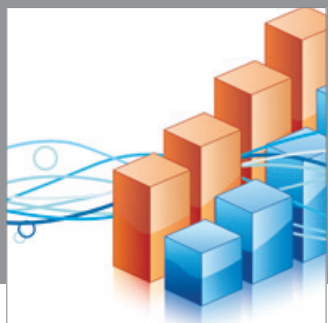

Advances in

Operations Research

mansans

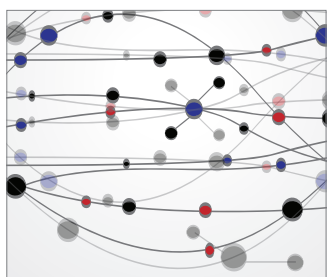

The Scientific World Journal
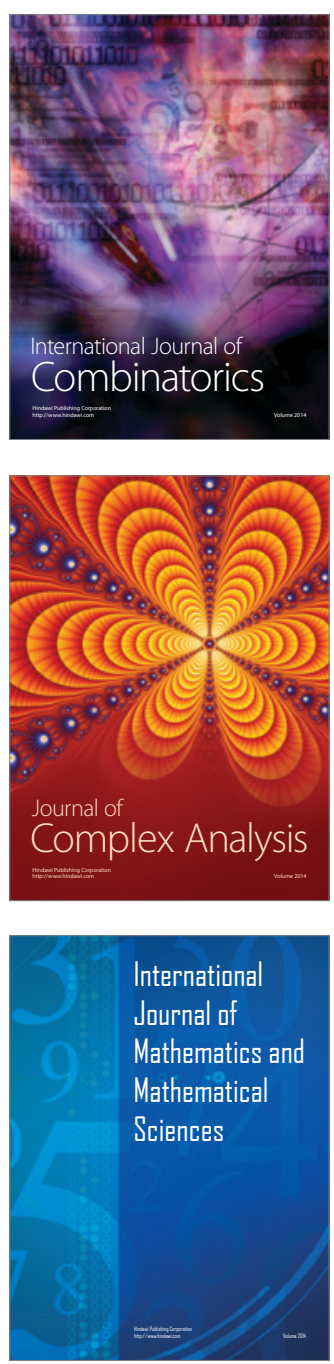
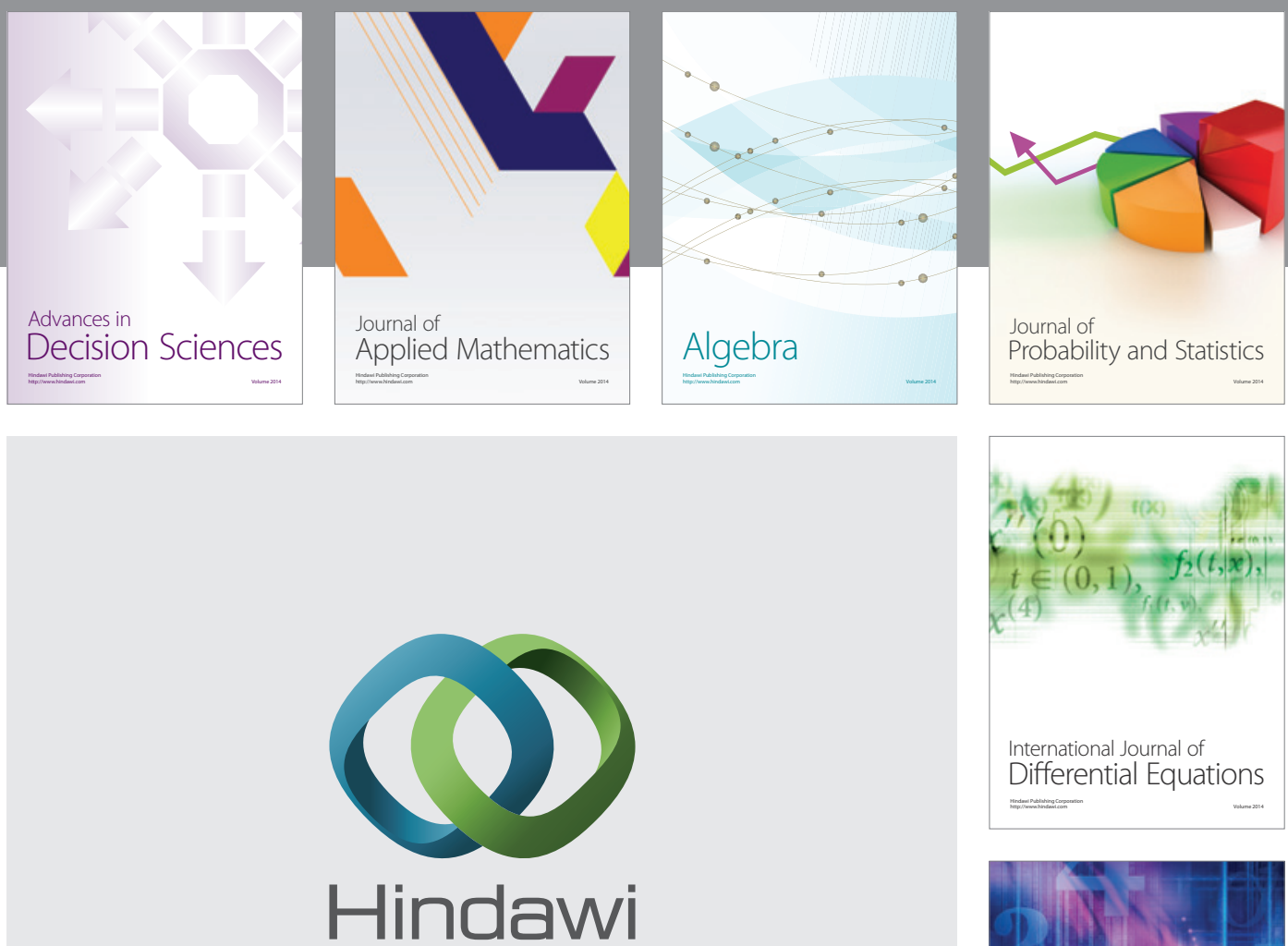

Submit your manuscripts at http://www.hindawi.com
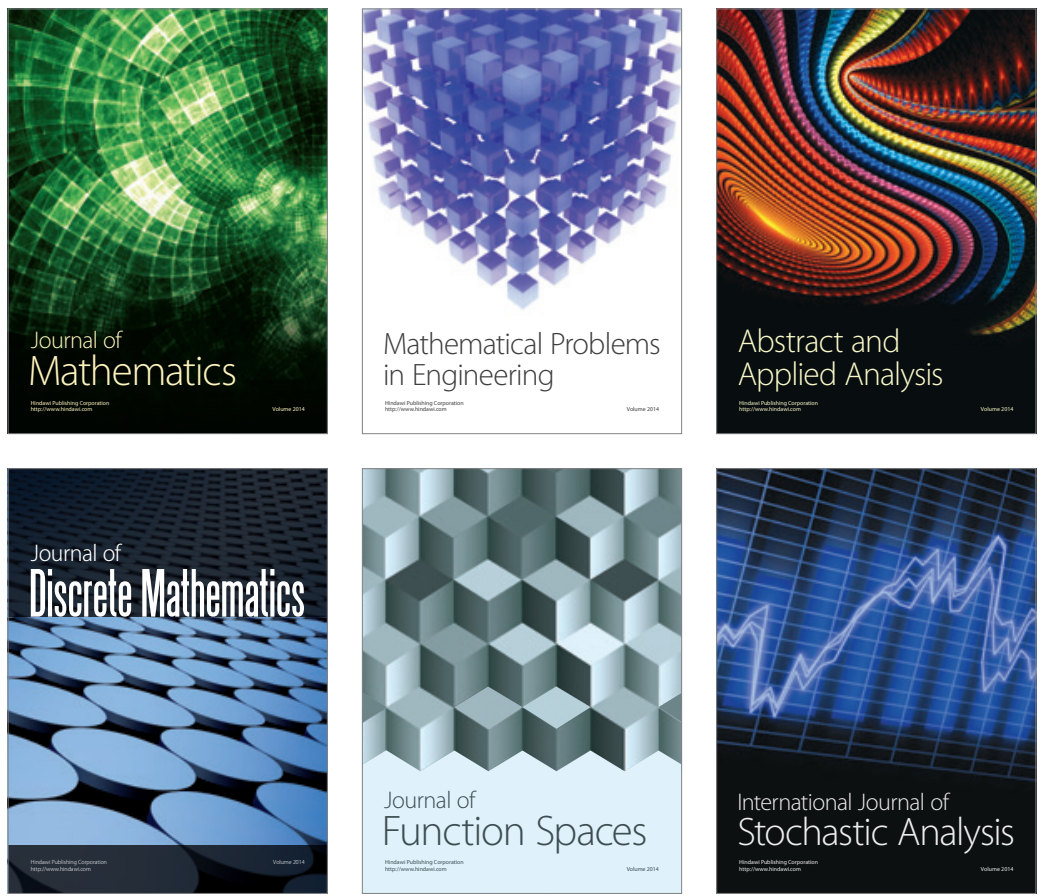

Journal of

Function Spaces

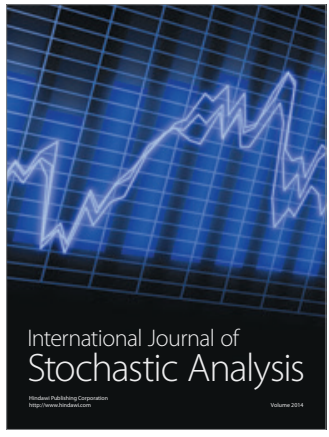

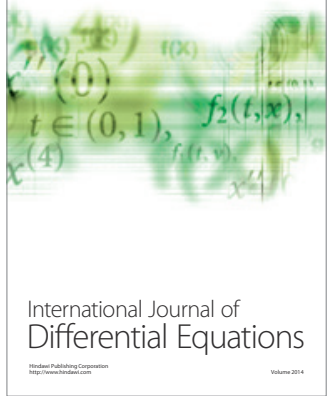
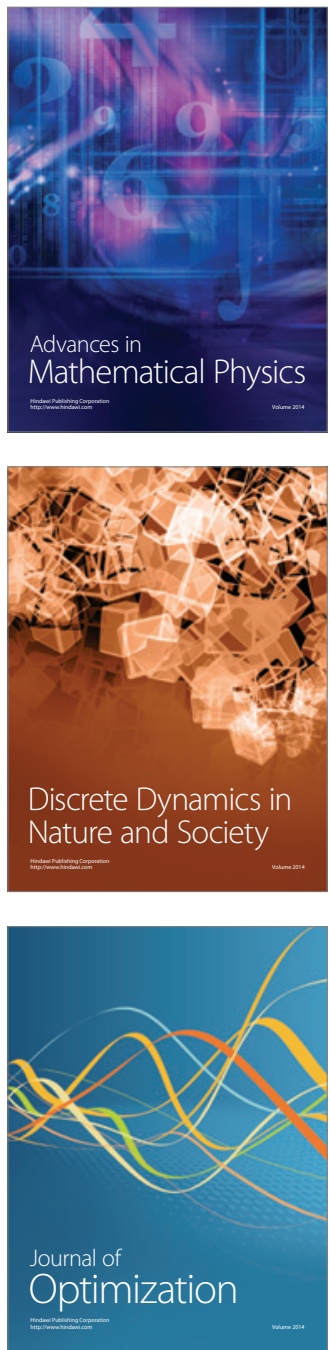